\title{
A Double-Sweeping Preconditioner for the Helmholtz Equation
}

\author{
Mehran Eslaminia $^{\mathrm{a}}$ and Murthy N. Guddati, ${ }^{\mathrm{b}, *}$ \\ ${ }^{a}$ Bentley Systems, Incorporated \\ 3850 North Causeway Blvd, Suite 1555 \\ Metairie, LA, 70002, USA \\ Email: mehran.eslaminia@bentley.com,Phone: (610)458-2697 \\ ${ }^{b}$ North Carolina State University \\ Department of Civil Engineering \\ 2501 Stinson Drive \\ Raleigh, NC 27695-7908, USA \\ Email:mnguddat@ncsu.edu,Phone:(919) 515-7699
}

Submission Date: July 15, 2015

Revision Date: January 24, 2016

* Corresponding Author. 


\begin{abstract}
ABSTARCT: A new preconditioner is developed to increase the efficiency of iterative solution of the Helmholtz equation. The key idea of the proposed preconditioner is to split the domain of interest into smaller subdomains and sequentially approximate the forward and backward components of the solution. The sequential solution is facilitated by approximate interface conditions that ignore the effect of multiple reflections. The efficiency of the proposed method is tested using various 2-D heterogeneous media. We observe that the proposed preconditioner results in good convergence, with number of iterations growing very slowly with increasing frequency. We also note that the mesh size and number of subdomains do not affect the convergence rate. Finally, we find that the overall computational time is much smaller than that of the sweeping preconditioner.
\end{abstract}

KEYWORDS: preconditioner, Helmholtz equation, iterative solver, amplitude-preserving propagator

\title{
1 Introduction
}

The Helmholtz and related equations commonly appears in the study of wave propagation in acoustic, elastic and electromagnetic media in wide ranging applications. Despite its widespread attention it received, Helmholtz equation at high frequencies still poses a significant computational challenge, due to the fine discretization leading to large linear systems. Often, these linear systems necessitate the use of iterative methods such as Krylov subspace methods, Domain Decomposition (DD) methods or a combination [1-3]. Unfortunately, these methods typically show very slow convergence rate for the Helmholtz problem, especially at high frequencies [4].

A preconditioner, if designed properly, could improve the convergence rate of Krylov methods. Over past two decades, various preconditioners have been introduced to solve the Helmholtz equation. In this paper, we focus primarily on preconditioners that involve sequential solution over subdomains in a prescribed direction, i.e. marching type preconditioners. It is worth noting that these class of preconditioners usually utilize high performance parallel algorithms for the solution within a given subdomain (e.g. see [5]). An 
extensive review can be found in $[4,6]$; these preconditioners can be classified into several groups. The first class is based on incomplete LU (ILU) factorization of the linear system using Gaussian elimination [7]. Although these methods yield good approximation, they eventually become computationally expensive for very large systems. A similar approach is to construct ILU approximation analytically - hence called Analytic ILU or AILU [8]. These methods are based on factorizing the Helmholtz operator into two nonlocal operators (forward and backward operators) and then approximating these operators using quadratic functions in Fourier domain, resulting in local operators. It has been shown that AILU methods are superior to classical ILU methods, but the number of iterations in Krylov solver still grow with increasing frequency [6]. The third group is called shifted Laplacian preconditioners where a modified Helmholtz equation is shifted by a complex value $[9,10]$. It has been shown that these methods can be good preconditioners but the number of iterations still grow relatively fast by increasing the frequency [6].

Recently, Engquist and Ying [11] developed a novel preconditioner based on perfectly matched layers, named the sweeping preconditioner. This method involves approximating Schur complement in $L D L^{T}$ with perfectly matched layer (PML). They have shown that the number of iterations grow relatively slowly with increasing frequency. More recently, a closely related method is proposed independently by Stolk [12] and Vion and Geuzaine [2] in the context of DD methods. This new approach utilizes an interface condition to reduce artificial reflection along subdomain interfaces, and a sequential doublesweeping scheme over the subdomains to solve an approximate solution, leading to improved convergence.

Our research in seismic migration and inversion led to the development of so-called amplitude-preserving propagators, which were instrumental in improving the convergence of both least-squares migration and full waveform inversion [13]. These propagators result in approximate solution to the Helmholtz equation through efficient sequential solution of downward and upward wavefield. Triggered by the similarities of this approach to the sweeping preconditioner idea, we investigate the application of this amplitude-preserving propagator as a preconditioner to the Helmholtz equation. The resulting method appears to be 
superior to the sweeping preconditioner, and comparable to Stolk's method. The remainder of the paper contains the details of the method as well as illustration of its effectiveness (this material as well as the work related to least-squares migration and full waveform inversion is also documented in [14].

This paper is organized as follows. We first introduce the proposed method for a 1-D model problem in section 2. We also compare our method with related works using a 1-D example. Next we present the extension to higher dimensions in section 3. Section 4 contains the accuracy of the proposed method as an approximate solver. In section 5, we show the performance of the proposed method as a preconditioner by carrying out multiple numerical simulations with different velocity profiles in 2-D; the efficiency of the method is also compared to the sweeping preconditioner. We conclude the paper with brief closing remarks in Section 6.

\section{1-D Formulation}

\subsection{Model Problem}

For simplicity, we first demonstrate the proposed approximate solver for a 1-D unbounded domain. Consider the time-harmonic wavefield $u$, due to the external force $f$, which is only non-zero within the domain of interest $\Omega \equiv(0,1)$. Field variable $u$ satisfies the Helmholtz equation:

$$
-\frac{\partial^{2} u}{\partial x^{2}}-\frac{\omega^{2}}{c^{2}} u=f(x) \text { for } x \in \Omega,
$$

where $\omega$ is the temporal frequency and $c$ is the wave velocity that varies only inside $\Omega$ and constant outside. Therefore appropriate absorbing boundary conditions can be applied on the boundaries, thus resulting in,

$$
\frac{\partial u}{\partial x}=\lambda u \text { on } x=0, \quad-\frac{\partial u}{\partial x}=\lambda u \text { on } x=1,
$$

where $\lambda$ is Dirichlet-to-Neumann (DtN) operator, which is essentially the stiffness of the homogeneous half-space, and is given by: 


$$
\left.\lambda\right|_{x=0}=-i \frac{\omega}{c(0)},\left.\quad \lambda\right|_{x=1}=-i \frac{\omega}{c(1)} .
$$

Note that while the above model is simple, the proposed method can be extended to higher dimension and complex problems as discussed in the subsequent sections.

\subsection{Approximate Solver}

The basic idea is to split the wavefield into right propagating component $u_{r}$ and left propagating component $u_{l}$, and approximate each of them by ignoring the multiple reflections. Thus, $u_{r}$ is the right propagating wavefield obtained by proper transmission of amplitude across material interfaces, while $u_{l}$ is the left propagating wavefield resulting from primary reflections at the same interfaces. With such an approximation, $u_{r}$ can be solved sequentially from left of the domain to the right. $u_{l}$ can then be solved from the right of the domain to the left. A critical aspect of this solution is to accurately obtain the transmission and reflection amplitudes. In what follows, we present the mathematical framework for solving for both $u_{r}$ and $u_{l}$.

For the sake of sequential solution, domain $\Omega$ is partitioned into smaller subdomains $\Omega^{j} \equiv\left(x^{j}, x^{j+1}\right)$ as shown in Figure 1. The boundaries of $\Omega^{j}$ are denoted by $\Gamma_{l}^{j}: x=x^{j}$ on the left and $\Gamma_{r}^{j}: x=x^{j+1}$ on the right. We use the superscript $j$ to represent entities associated with $\Omega^{j}$, and subscripts $l$ and $r$ are used to represent the traces of variables on the left and right respectively. With such notation, the boundary value problem associated with $\Omega^{j}$ can be written as:

$$
\begin{gathered}
-\frac{\partial^{2} u^{j}}{\partial x^{2}}-\frac{\omega^{2}}{c^{2}} u^{j}=f \quad \text { in } \Omega^{j}, \\
u_{l}^{j}=u_{r}^{j-1},\left.\quad \frac{\partial u^{j}}{\partial x}\right|_{x^{j}}=\left.\frac{\partial u^{j-1}}{\partial x}\right|_{x^{j}}, \\
u_{r}^{j}=u_{l}^{j+1},\left.\quad \frac{\partial u^{j}}{\partial x}\right|_{x^{j+1}}=\left.\frac{\partial u^{j+1}}{\partial x}\right|_{x^{j+1}} .
\end{gathered}
$$


The equations in (5) represent the continuity of the wavefield and traction across the interfaces and cause the solution to be coupled across all the subdomains. The basic idea of the proposed method is to approximate the interface conditions (5) that relaxes the coupling and facilitates sequential solution, which is performed in two steps. First the right propagating wavefield, $u_{r}$, is computed considering only right transmission at the interfaces. The wavefield is then corrected by adding the left propagating wavefield, $u_{l}$, resulting from the primary reflections at all the interfaces. The details are discussed below.

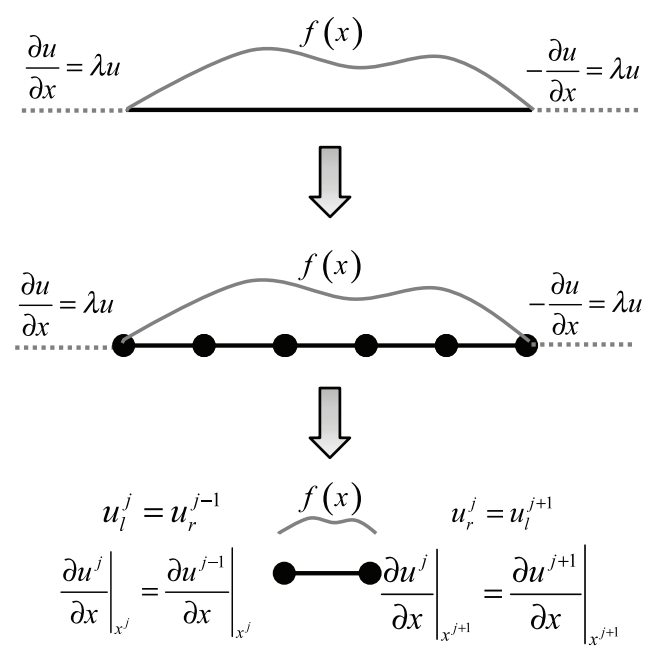

Figure 1. Schematic solution for 1-D problems

\subsection{Right Propagation Wavefield}

The right propagating wavefield $u_{r}$ is determined by solving the sub-problems from left to right, starting with the first subdomain. Thus, we compute the wavefield in $j^{\text {th }}$ subdomain, from the wavefield in $j-1^{\text {th }}$ subdomain. This is achieved by applying the following conditions:

1. The incoming wavefield from $j-1$ subdomain must be propagated into the current subdomain without any reflection at the left interface. 
2. The change in material properties (e.g. wave velocity) must be considered to get accurate transmission of wavefield at the right interface (and reflection, which will be used for left propagation in the next subsection).

Condition 1 is enforced by adding a half-space at the left interface, with material properties matching with the left boundary $\Gamma_{l}^{j}: x=x^{j}$. Condition 2 is enforced by adding another half-space at the right interface, but with material properties consistent with left boundary of next subdomain $\Gamma_{l}^{j+1}: x=x^{j+1}$ (to obtain the appropriate reflection and transmission at the interface). Note that if the half-space is matched with the right boundary of current subdomain $\Gamma_{r}^{j}: x=x^{j+1}$, there will be no reflection and the transmission will be inaccurate, and we would not get the desired right propagating wavefield.

Mathematically, attaching the right half-space translates to,

$$
\frac{\partial u_{r}^{j}}{\partial x}+\lambda_{l}^{j+1} u_{r}^{j}=0 \text { on } \Gamma_{r}^{j},
$$

where $\lambda_{l}^{j+1}$ is the stiffness of half-space matching with the left of the next subdomain. This is in contrast with the right interface conditions in (5) - we no longer need the solution of the right subdomain to obtain the solution in the current subdomain, which is appropriate given that we are focusing only on the right propagating wavefield.

The boundary condition on the left is slightly more involved; in addition to attaching a half-space, we have incident wave coming from the previous subdomain, which is the right propagating wavefield at the right boundary of previous subdomain $\Gamma_{r}^{j-1}: x=x^{j}$. The left half-space can be modeled using half-space stiffness as in Equation (6), while the incident wave is modeled using standard scattering formalism [15]:

$$
-\left.\frac{\partial u_{r}^{j}}{\partial x}\right|_{x^{j}}+\lambda_{l}^{j} u_{r, l}^{j}=-\left.\frac{\partial u_{r}^{j-1}}{\partial x}\right|_{x^{j}}+\lambda_{l}^{j} u_{r, r}^{j-1}
$$

where $u_{r, l}^{j}$ and $u_{r, r}^{j-1}$ are right propagating wavefield at the left of $j^{\text {th }}$ subdomain right propagating wavefield at the right of $j-1^{\text {th }}$ subdomain, respectively. 
Using Equation (6) from previous subdomain, we have,

$$
\left.\frac{\partial u_{r}^{j-1}}{\partial x}\right|_{x^{j}}=-\lambda_{l}^{j} u_{r, r}^{j-1}
$$

and Equation (7) simplifies to:

$$
-\frac{\partial u_{r}^{j}}{\partial x}+\lambda_{l}^{j} u_{r}^{j}=2 \lambda_{l}^{j} u_{r, r}^{j-1} \text { on } \Gamma_{l}^{j} .
$$

In summary, the right propagating wavefield can be obtained by solving the local boundary value problem:

$$
\begin{aligned}
& -\frac{\partial^{2} u_{r}^{j}}{\partial x^{2}}-\frac{\omega^{2}}{c(x)^{2}} u_{r}^{j}=f(x) \text { in } \Omega^{j}, \\
& -\frac{\partial u_{r}^{j}}{\partial x}+\lambda_{l}^{j} u_{r}^{j}=2 \lambda_{l}^{j} u_{r, r}^{j-1} \text { on } \Gamma_{l}^{j}: x=x^{j}, \\
& \frac{\partial u_{r}^{j}}{\partial x}+\lambda_{l}^{j+1} u_{r}^{j}=0 \text { on } \Gamma_{r}^{j}: x=x^{j+1} .
\end{aligned}
$$

Using finite difference or finite element method, the discrete sub-problem set corresponding to Equation (10) is

$$
\left[\begin{array}{ll}
A^{j} & B^{j} \\
B^{j} & C^{j}
\end{array}\right]\left\{\begin{array}{l}
u_{r, l}^{j} \\
u_{r, r}^{j}
\end{array}\right\}=\left\{\begin{array}{l}
f_{l}^{j} \\
0
\end{array}\right\}+\left\{\begin{array}{c}
-\left.\frac{\partial u_{r}^{j}}{\partial x}\right|_{\Gamma_{l}^{j}} \\
\left.\frac{\partial u_{r}^{j}}{\partial x}\right|_{\Gamma_{r}^{j}}
\end{array}\right\}
$$

where $A^{j}, B^{j}$, and $C^{j}$ are matrix elements corresponding to the discretization, where we condense out the interior nodes for the sake of simple presentation. At each step of the solution, the effect of external forces is only applied on the left boundary of subdomain. Thus, we do not need to consider the external force on the right boundary since it will be captured in the next step of the solution. The traction terms on the right hand side are determined by boundary conditions given in Equation (10). Therefore we get,

$$
\left[\begin{array}{ll}
A^{j} & B^{j} \\
B^{j} & C^{j}
\end{array}\right]\left\{\begin{array}{l}
u_{r, l}^{j} \\
u_{r, r}^{j}
\end{array}\right\}=\left\{\begin{array}{c}
f_{l}^{j} \\
0
\end{array}\right\}+\left\{\begin{array}{c}
-\lambda_{l}^{j} u_{r, l}^{j}+2 \lambda_{l}^{j} u_{r, r}^{j-1} \\
-\lambda_{r}^{j+1} u_{r, r}^{j}
\end{array}\right\},
$$


which is rewritten as:

$$
\left[\begin{array}{cc}
A^{j}+\lambda_{l}^{j} & B^{j} \\
B^{j} & C^{j}+\lambda_{r}^{j+1}
\end{array}\right]\left\{\begin{array}{c}
u_{r, l}^{j} \\
u_{r, r}^{j}
\end{array}\right\}=\left\{\begin{array}{c}
f_{l}^{j} \\
0
\end{array}\right\}+\left\{\begin{array}{c}
2 \lambda_{l}^{j} u_{r, r}^{j-1} \\
0
\end{array}\right\} .
$$

\subsection{Left Propagating Wavefield}

The body force and material discontinuities cause left propagating wavefield that must be added to the right propagating wavefield - left propagation step discussed in this section essentially captures this effect of primary reflections and external forces. In the absence of external forces, the reflection is simply the difference between the transmitted wave (right propagating wavefield at the left of $j+1^{\text {th }}$ subdomain $u_{r, l}^{j+1}$ ) and the incident wave (right propagating wavefield at the right of $j^{\text {th }}$ subdomain $u_{r, r}^{j}$ ). This reflected wavefield should be added to the left propagating wavefield $u_{l, l}^{j+1}$ from the right subdomain to obtain the total incident wave field for $j^{\text {th }}$ subdomain:

$$
u_{r, l}^{j+1}-u_{r, r}^{j}+u_{l, l}^{j+1}
$$

When we consider the effects of body forces in the computation of right propagating wave field, $u_{r, l}^{j+1}$ is the combination of the transmitted wavefield as well as the left propagating wavefield due to the body forces in $j+1^{\text {th }}$ subdomains. Thus, the difference $u_{r, l}^{j+1}-u_{r, r}^{j}$ in this case is the combination of reflected wavefield and left propagating wavefield due to body forces. Since the effect of body forces on left propagating wavefield is already captured through this term, we should no longer consider the source term explicitly. Hence, we use the term in Equation (14) for the incident wavefield and explicitly make the body force zero.

Once the incident wavefield is determined according to Equation (14), the solution procedure is similar to the previous subsection. Following Equation (10), the left propagating sub-problem for subdomain $j$ is written as: 


$$
\begin{aligned}
& -\frac{\partial^{2} u_{l}^{j}}{\partial x^{2}}-\frac{\omega^{2}}{c(x)^{2}} u_{l}^{j}=0 \text { in } \Omega^{j}, \\
& -\frac{\partial u_{l}^{j}}{\partial x}+\lambda_{r}^{j-1} u_{l}^{j}=0 \text { on } \Gamma_{l}^{j}: x=x^{j}, \\
& \frac{\partial u_{l}^{j}}{\partial x}+\lambda_{r}^{j} u_{l}^{j}=2 \lambda_{r}^{j}\left(u_{r, l}^{j+1}-u_{r, r}^{j}+u_{l, l}^{j+1}\right) \text { on } \Gamma_{r}^{j}: x=x^{j+1} .
\end{aligned}
$$

We re-emphasize that the external body force is zero for left sweeping since its effects are already considered as part of the incident wavefield in the scattering boundary condition in right sweeping. After appropriate discretization of the boundary value problem in Equation (15), we get a discrete system similar to Equation (13):

$$
\left[\begin{array}{cc}
A^{j}+\lambda_{r}^{j-1} & B^{j} \\
B^{j} & C^{j}+\lambda_{r}^{j}
\end{array}\right]\left\{\begin{array}{l}
u_{l, l}^{j} \\
u_{l, r}^{j}
\end{array}\right\}=\left\{\begin{array}{c}
0 \\
2 \lambda_{r}^{j}\left(u_{r, l}^{j+1}-u_{r, r}^{j}+u_{l, l}^{j+1}\right)
\end{array}\right\} .
$$

\section{$2.5 \quad$ 1-D Algorithm and Analysis}

The computation of wave field using proposed approximate solver can be summarized in the following algorithm:

Algorithm 1: 1-D Double-Sweeping Solver

1. $u_{r, l}^{0}=0$

2. for $j=1$ to $n$ Solve: Equation (13)

3. $u_{l, l}^{n}=0$

4. for $j=n-1$ to 1

a. Compute: the left incident wavefield $u_{r, l}^{j+1}-u_{r, r}^{j}+u_{l, l}^{j+1}$

b. Solve: Equation (16)

5. Compute: wave field: $u=u_{r}+u_{l}$

We test the accuracy of the algorithm for 1-D wave propagation with the following wave velocity:

$$
c(x)=\left\{\begin{array}{cc}
2 & 0.4 \leq x \leq 0.6 \\
1 & \text { otherwise }
\end{array} .\right.
$$


The domain of interest is $\Omega \equiv(0,1)$, which is discretized with 1000 linear finite elements. The response is computed due to a point source at $x=0.15$. We used 20 subdomains to get approximate solution which is compared with true solution at frequency $10 \mathrm{~Hz}(\omega=20 \pi)$ in Figure 2. Given the fine discretization, the only difference between the solutions can be attributed to the multiple reflections which are not captured in the proposed method.

We also test the accuracy of the proposed method in the time domain, by taking inverse Fourier transform of the frequency-domain solutions. We consider the above velocity model and obtain the wavefield for the frequency range 0.2 to $100 \mathrm{~Hz}$ with a $0.2 \mathrm{~Hz}$ interval. The source excitation is a Ricker pulse with central frequency of $25 \mathrm{~Hz}$ located at 0.15 . Snapshots of the true and approximate solutions are compared in Figure 3. This comparison shows that the proposed method computes the forward propagating wavefield and primary reflections to a high level of accuracy and the only difference is the effect of multiple reflections.

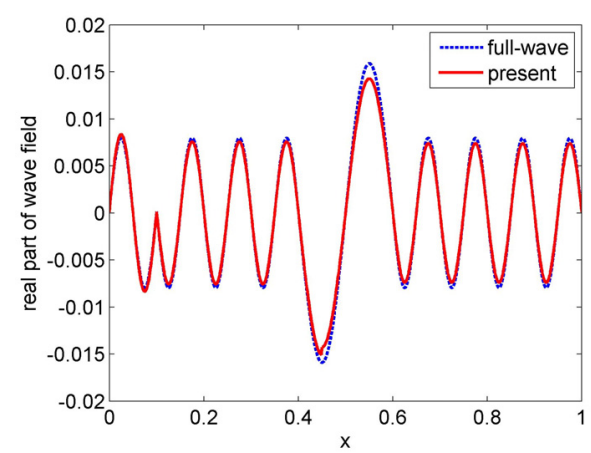

(a)

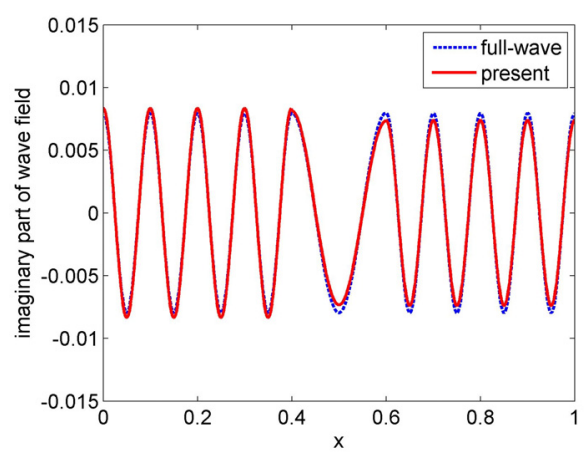

(b)

Figure 2. Comparison between true and approximate solutions in 1-D medium. a) real part and b) imaginary part. 


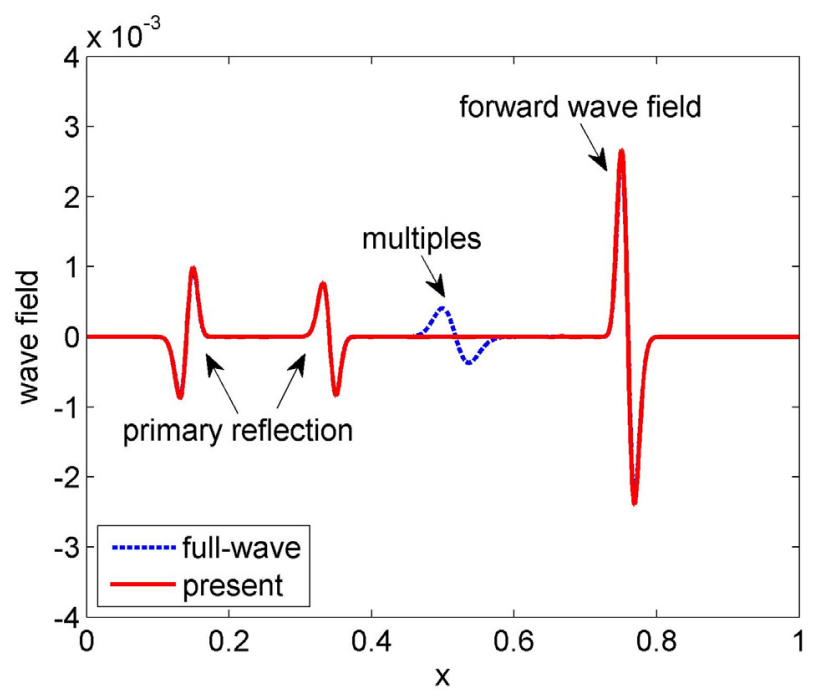

Figure 3. Comparison of true and approximate solutions at $t=0.6 \mathrm{~s}$

\subsection{Related Works}

In this section, we compare the present method with similar work - sweeping preconditioner [11] and double-sweep domain-decomposition (DD) method proposed by Stolk [12]. In particular, the approximate wavefield is determined using each method for the 1-D problem defined in Section 2.5. The results in the frequency domain are shown in Figure 4 and 5 and the time-domain solutions are shown in Figure 6 and 7. Comparison of these solutions and those from the proposed method in Figure 2 and 3, indicates that the solution of sweeping preconditioner is not very accurate, while Stolk's method has better accuracy. In addition, our approach seems to be slightly better than Stolk's method for this particular problem, but in general expected to be comparable. 


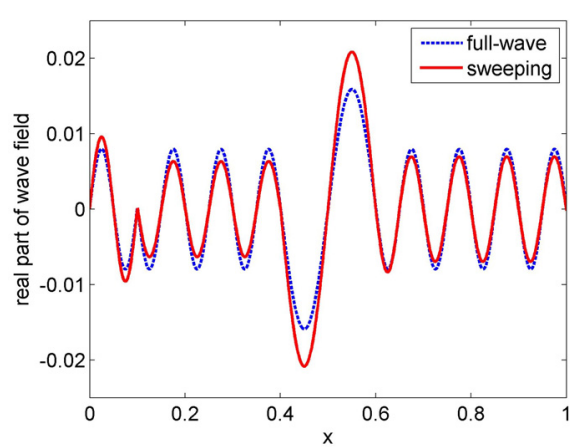

(a)

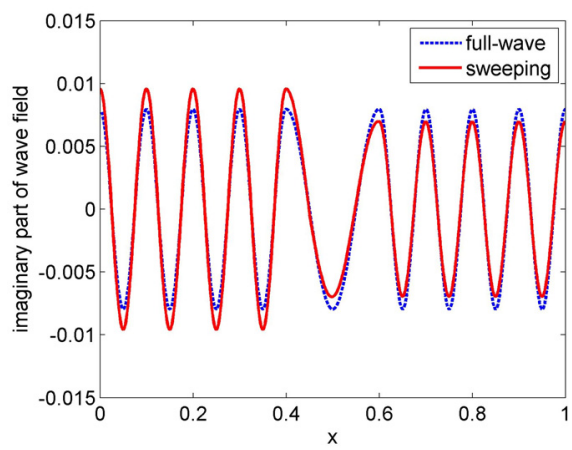

(b)

Figure 4. Comparison true and approximate solutions for sweeping preconditioner [11]. a) real part and b) imaginary part.

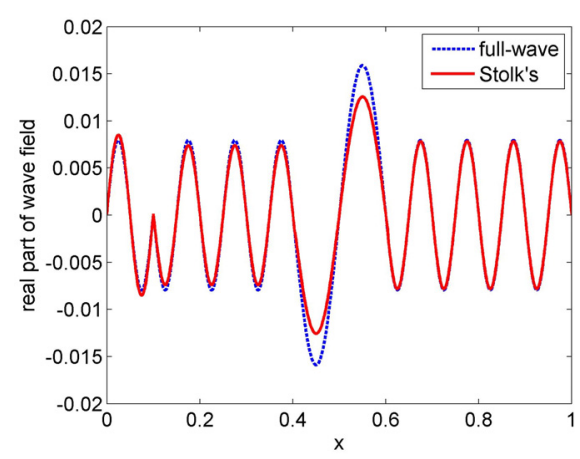

(a)

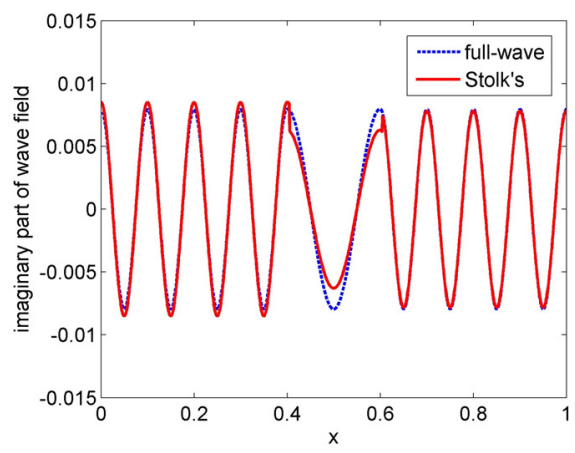

(b)

Figure 5. Comparison true and approximate solutions for double-sweep method by Stolk [12]. a) real part and b) imaginary part. 


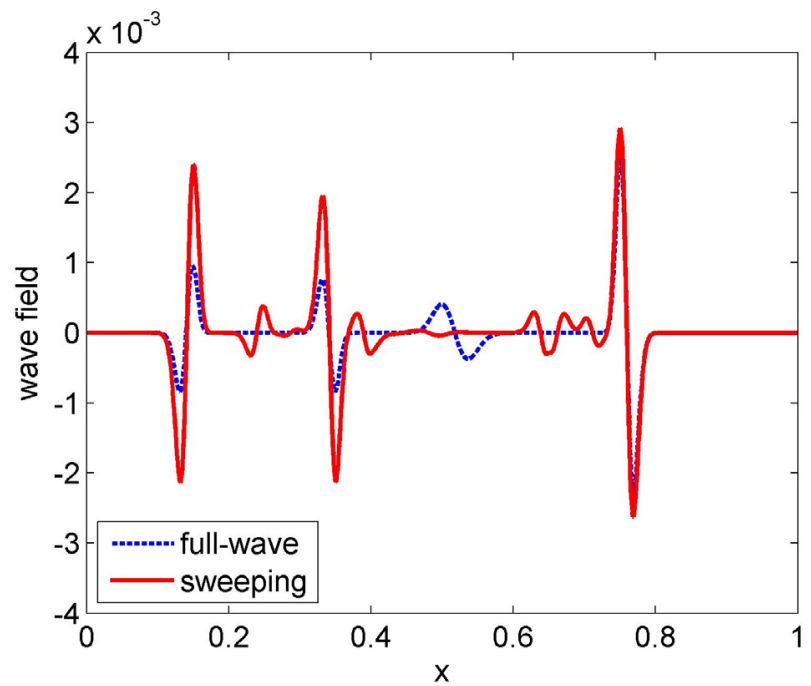

Figure 6. Comparison of true and approximate solutions at $t=0.6 \mathrm{~s}$ for sweeping preconditioner [11].

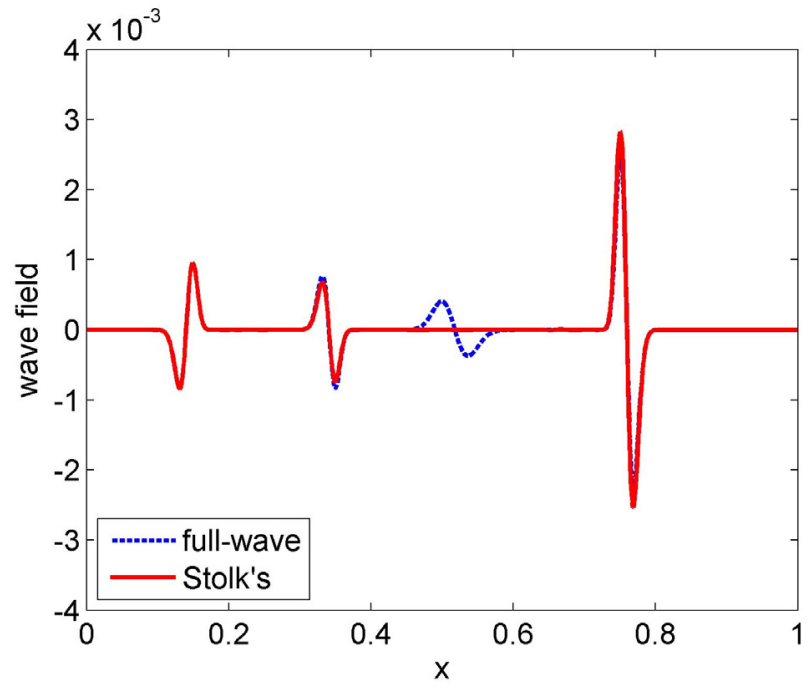

Figure 7. Comparison of true and approximate solutions at $t=0.6 \mathrm{~s}$ for double-sweep method by Stolk [12]. 
The similarity in accuracy between our method and Stolk's method is not surprising as they share many common aspects, but there are also some differences:

- The boundary condition for outgoing wavefield: The most notable difference is the approximate interface condition on right interface for right propagation and left interface for left propagation. In our method, we use modified radiation condition to get accurately transmitted wave field, where as Sotlk's method approximates the outgoing wavefield using an unaltered absorbing condition corresponding to the current subdomain.

- Backward propagation: The second difference is computing backward propagation. In the present method, we directly use the reflection on the interfaces to get backpropagating wavefield. We also use the same subdomains, albeit with different interface conditions, to get forward and backward wavefield. Stolk uses staggered subdomains for backward sweeping, to move the force (residual) into the subdomain.

- Computational cost: Both methods require factorization of local wave operators with similar boundary conditions. Therefore, their computational complexity and cost are similar provided that the same treatment is used for modeling the half-space. Unlike in Stolk's method that uses PML, we propose the use of PMDL, which is shown to be superior to PML due to midpoint integration [16]. The benefits of midpoint integration in the context of our formulation is clearly illustrated in Section 5.1.3. Use of PMDL in place of PML in Stolk's method may also result in similar benefits.

- Accuracy: The proposed method and Stolk's method are comparable in accuracy and we expect both methods to perform similarly as preconditioners.

- Parallelization: The present method and Stolk's method are both sequential solvers. However, they may benefits from parallel algorithms (e.g. the parallel multi-frontal algorithm proposed in [5]) to factorize the linear system corresponding a given subdomain. 


\subsection{GMRES Convergence for 1-D domain}

Consider the linear system corresponding to model problem in Equation (1),

$$
\mathbf{S u}=\mathbf{f} .
$$

The left-preconditioned system can be defined by:

$$
\mathbf{P}^{-1} \mathbf{S u}=\mathbf{P}^{-1} \mathbf{f}
$$

where $\mathbf{P}^{-1}$ is the preconditioner defined by the approximate solver in Algorithm 2.1. We compare GMRES convergence rate using sweeping preconditioner, Stolk's method, and the proposed method. We use the 1-D velocity model defined in Section 2.5 and the excitation is a unit point force positioned at $x=0.5$. The thickness of each subdomain is 12 finite elements.

The numbers of GMRES iterations are compared in Table 1 for various frequencies considering a relative error of 1e-6s. The convergence behaviors are also compared for frequencies $16 \mathrm{~Hz}$ and $256 \mathrm{~Hz}$ in Figure 8. These results show that, as expected, the performance of the proposed method is very similar to Stolk's method. However, both methods show faster convergence compared to the sweeping preconditioner, especially at high frequencies; the sweeping preconditioner gradually loses its effectiveness with increasing frequency.

Table 1. Comparison of iteration counts using different preconditioner in 1-D domain

\begin{tabular}{ccccc}
\hline $\begin{array}{c}\text { Frequency } \\
(\mathrm{Hz})\end{array}$ & Number of elements & Sweeping & Stolk & present \\
\hline 8 & 64 & 5 & 4 & 4 \\
16 & 128 & 5 & 4 & 4 \\
32 & 256 & 7 & 4 & 4 \\
64 & 512 & 8 & 4 & 5 \\
128 & 1024 & 10 & 4 & 4 \\
256 & 2048 & 13 & 5 & 5 \\
\hline
\end{tabular}




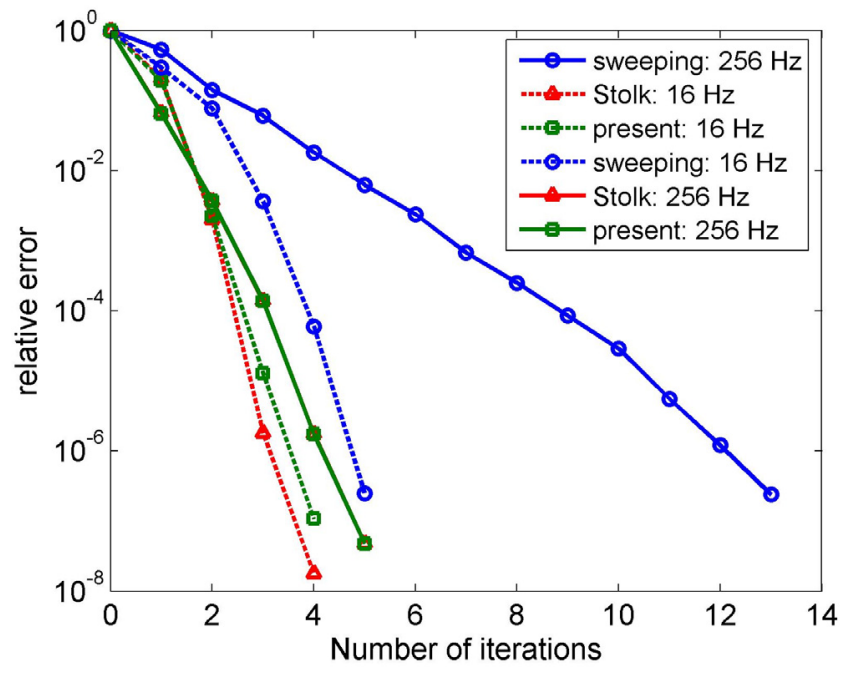

Figure 8. Comparison of GMRES convergence rates using different preconditioners.

It is well-known that the convergence rate of GMRES method is a function of eigenvalues of to the linear system [7]. A linear system with a wide eigenvalue spectrum shows slow convergences while a system with clustered eigenvalues requires small number of GMRES iterations. We compare the eigenvalues corresponding to the preconditioned linear system in Equation (19) using sweeping preconditioner, Stolk's DD method, and the present method. The eigenvalue amplitudes of the preconditioned systems are plotted in Figure 9 for various frequencies. These results show that the spectrum corresponding to the sweeping preconditioner becomes wider with increasing frequency which in turn results in slower convergence of GMRES. On the other hand, the eigenvalues of Stolk's method and the present preconditioner remain clustered for all frequencies. Therefore, the number of iterations required for convergence does not change with increasing frequency as numerically verified in Table 1. 


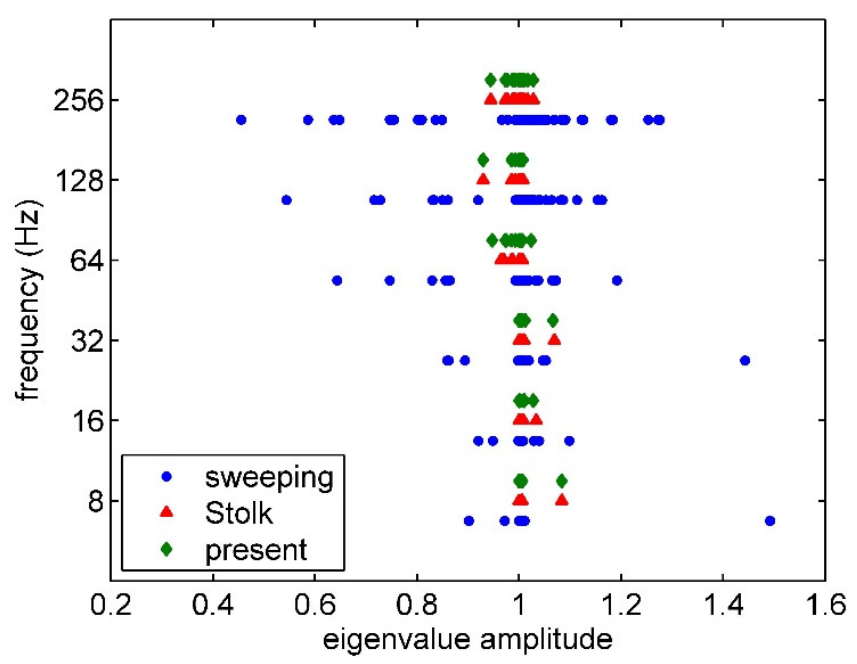

Figure 9. Eigenvalues spectrum of the preconditioned system $\mathbf{P}^{-1} \mathbf{S}$ using different preconditioners.

\section{Extension to Higher Dimensions}

Extension of the proposed method to higher dimensions is conceptually straightforward, but the details are a bit more involved (e.g. DtN maps). This section considers following Helmholtz equation for the domain of interest $\Omega \equiv\left(\begin{array}{ll}0 & 1\end{array}\right)^{n}$ for $n=1,2$, or 3 ,

$$
-\nabla^{2} u-\frac{\omega^{2}}{c(\mathbf{x})^{2}} u=f(\mathbf{x}) \text { for } \mathbf{x} \in \Omega
$$

where $\omega$ is the temporal (angular) frequency and $c$ is the wave velocity. The absorbing boundary conditions are applicable on the boundaries $\partial \Omega$ :

$$
-\frac{\partial u}{\partial \mathbf{n}}=\lambda u
$$

where DtN map $\lambda$ is a spatially nonlocal operator, and $\mathbf{n}$ is a unit outward normal vector. The exact DtN map can be written formally for 2-D problem with the boundary parallel to the $x$ axis as, 


$$
\lambda=-i \sqrt{\frac{\partial^{2}}{\partial x^{2}}-\frac{\omega^{2}}{c^{2}}} .
$$

The above DtN map can be approximated using a local absorbing boundary condition (ABC) such as rational ABCs [17-19], perfectly matched layer (PML) [20, 21], and the more recently developed perfectly matched discrete layer (PMDL) [22, 23]. Details are discussed in Appendix A.

Similar to the 1-D case, the domain is first divided to smaller subdomains. While it is possible to partition the domain in any direction, we choose $n$ horizontal slabs along the $x$ axis, represented by $\Omega^{j}$ for $j=1, \ldots, n$. The boundaries of $\Omega^{j}$ are denoted by $\Gamma_{t}^{j}$ at the top and $\Gamma_{b}^{j}$ at the bottom. We use subscripts $t$ and $b$ to represent the traces of variables at top and bottom, respectively. The approximate solution is determined in two steps. First, the downward propagating (along the positive $z$-axis) wavefield $u_{d}$, is computed. The wavefield is then corrected by adding the upward (reflected) wavefield $u_{u}$. Following the 1-D formulation and without going into the details, the sub-problems for downward and upward propagation of $j^{\text {th }}$ slab can be formally written as:

$$
\begin{aligned}
& -\nabla^{2} u_{d}^{j}-\frac{\omega^{2}}{c(\mathbf{x})^{2}} u_{d}^{j}=f(\mathbf{x}) \text { for } \mathbf{x} \in \Omega_{j}, \\
& \frac{\partial u_{d}^{j}}{\partial \mathbf{n}}+\lambda_{t}^{j} u_{d}^{j}=2 \lambda_{t}^{j} u_{d, b}^{j-1} \text { on } \Gamma_{t}^{j}, \\
& \frac{\partial u_{d}^{j}}{\partial \mathbf{n}}+\lambda_{t}^{j+1} u_{d}^{j}=0 \text { on } \Gamma_{b}^{j},
\end{aligned}
$$

and

$$
\begin{aligned}
& -\nabla^{2} u_{u}^{j}-\frac{\omega^{2}}{c(\mathbf{x})^{2}} u_{u}^{j}=0 \text { for } \mathbf{x} \in \Omega_{j}, \\
& \frac{\partial u_{u}^{j}}{\partial \mathbf{n}}+\lambda_{b}^{j-1} u_{u}^{j}=0 \text { on } \Gamma_{t}^{j}, \\
& \frac{\partial u_{u}^{j}}{\partial \mathbf{n}}+\lambda_{b}^{j} u_{u}^{j}=2 \lambda_{b}^{j}\left(u_{d, t}^{j+1}-u_{d, b}^{j}+u_{u, t}^{j+1}\right) \text { on } \Gamma_{b}^{j} .
\end{aligned}
$$


In above expressions, $u_{d, b}^{j}$ is downward propagating wavefield at the bottom of the $j^{\text {th }}$ subdomain, $u_{d, t}^{j+1}$ is downward propagating wavefield at the top of the $j+1^{\text {th }}$ subdomain, and $u_{d, t}^{j+1}$ is upward propagating wavefield at the top of the $j+1^{\text {th }}$ subdomain. The schematic for each sub-problem is presented in Figure 10 and 11.

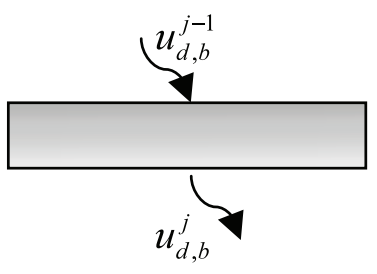

(a)

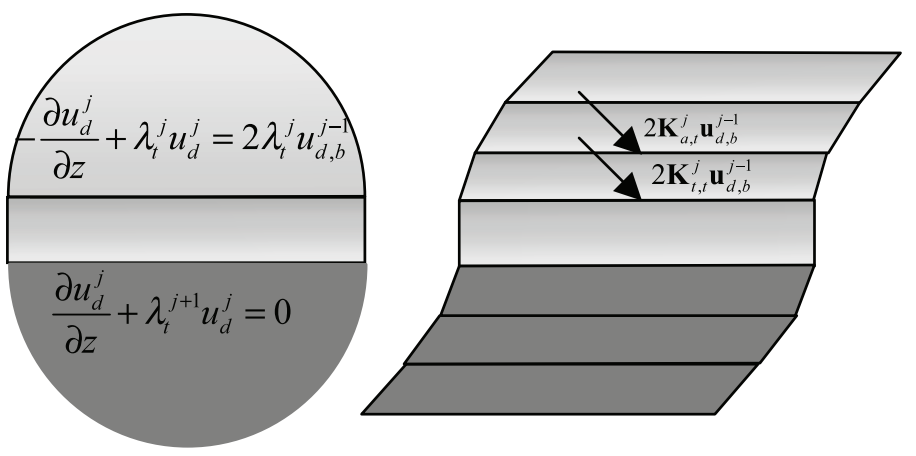

(b) (c)

Figure 10. Downward propagating framework. a) sub-problem schematic, b) sub-problem with approximate interface conditions, and c) discretized sub-problem.

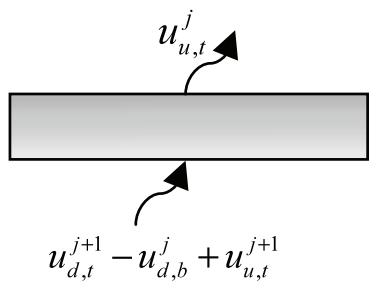

(a)

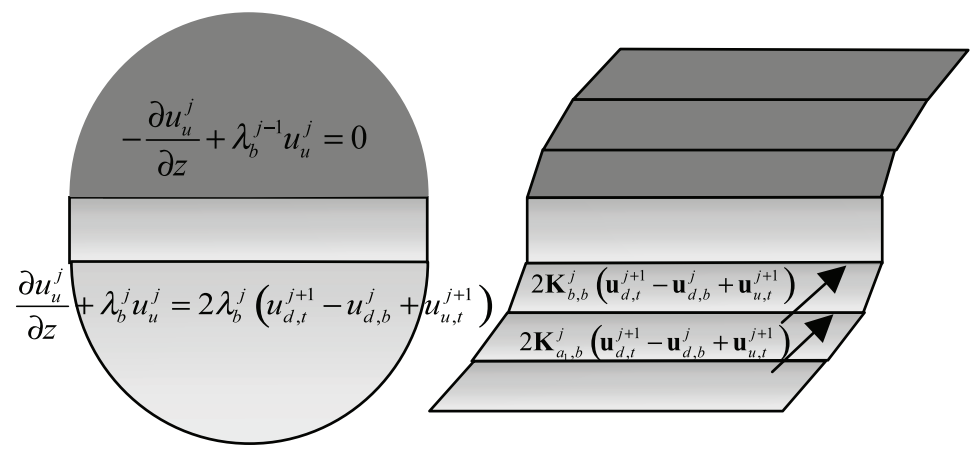

(b) (c)

Figure 11. Upward propagating framework. a) sub-problem schematic, b) sub-problem with approximate interface conditions, and c) discretized sub-problem. 


\subsection{Matrix Formulation}

Sub-problems in (23) and (24) are discretized using a finite difference or a finite element method. The first (downward propagating) sub-problem results in the following linear system:

$$
\left[\begin{array}{lll}
\mathbf{S}_{t, t}^{j} & \mathbf{S}_{t, i n}^{j} & \\
\mathbf{S}_{i n, t}^{j} & \mathbf{S}_{i n, i n}^{j} & \mathbf{S}_{i n, b}^{j} \\
& \mathbf{S}_{b, i n}^{j} & \mathbf{S}_{b, b}^{j}
\end{array}\right]\left\{\begin{array}{l}
\mathbf{u}_{d, t}^{j} \\
\mathbf{u}_{d, i n}^{j} \\
\mathbf{u}_{d, b}^{j}
\end{array}\right\}=\left\{\begin{array}{l}
\mathbf{f}_{t}^{j} \\
\mathbf{f}_{i n}^{j} \\
\mathbf{0}
\end{array}\right\}+\left\{\begin{array}{c}
\partial \mathbf{u}_{d}^{j} /\left.\partial \mathbf{n}\right|_{\Gamma_{t}^{j}} \\
\mathbf{0} \\
\partial \mathbf{u}_{d}^{j} /\left.\partial \mathbf{n}\right|_{\Gamma_{b}^{j}}
\end{array}\right\}
$$

where subscripts $t, b$, and in respectively denote top, bottom and interior grid points in the discretization, $\mathbf{S}_{m, n}^{j}$ are the matrix components corresponding to finite difference or finite element formulation, and $\mathbf{f}_{x}^{j}$ are the external force vectors. At each step of the solution, the effect of external forces is only applied at the top and interior nodes. Thus, we do not need to consider the external force on the bottom boundary since it will be captured in next step of the solution.

The traction terms on the right hand side are determined by boundary conditions given in Equation (23). These terms can be then approximated by a PML-like local absorbing boundary condition discussed in Appendix A. Therefore, the traction at the bottom interface can be approximated as (i.e. Equation (A-17))

$$
\left\{\begin{array}{c}
\partial \mathbf{u}_{d}^{j} /\left.\partial \mathbf{n}\right|_{\Gamma_{b}^{j}} \\
\mathbf{0} \\
\mathbf{0}
\end{array}\right\} \approx-\left[\begin{array}{lll}
\mathbf{K}_{t, t}^{j+1} & \mathbf{K}_{t, a_{1}}^{j+1} & \\
\mathbf{K}_{t, a_{1}}^{j+1} & \mathbf{K}_{a_{1}, a_{1}}^{j+1} & \mathbf{K}_{a_{1}, a}^{j+1} \\
& \mathbf{K}_{a, a_{1}}^{j+1} & \mathbf{K}_{a, a}^{j+1}
\end{array}\right]\left\{\begin{array}{l}
\mathbf{u}_{d, b}^{j} \\
\mathbf{u}_{d, b a_{1}}^{j} \\
\mathbf{u}_{d, b a}^{j}
\end{array}\right\}
$$

in which $\mathbf{u}_{d, b a_{1}}^{j}$ is the displacement vector corresponding to the auxiliary variables next to the bottom interface, $\mathbf{u}_{d, b a}^{j}$ are remaining auxiliary variables, and $\mathbf{K}_{m, n}^{j+1}$ are matrix elements to approximate $\lambda_{t}^{j+1}$ (corresponding to the top boundary of next slab). The traction at the top is similarly determined by approximating $\lambda_{t}^{j}$ using: 


$$
\left\{\begin{array}{c}
\partial \mathbf{u}_{d}^{j} /\left.\partial \mathbf{n}\right|_{\Gamma_{t}^{j}} \\
\mathbf{0} \\
\mathbf{0}
\end{array}\right\} \approx\left[\begin{array}{lll}
\mathbf{K}_{t, t}^{j} & \mathbf{K}_{t, a_{1}}^{j} & \\
\mathbf{K}_{t, a_{1}}^{j} & \mathbf{K}_{a, a_{1}}^{j} & \mathbf{K}_{a_{1}, a}^{j} \\
& \mathbf{K}_{a, a_{1}}^{j} & \mathbf{K}_{a, a}^{j}
\end{array}\right]\left\{-\left\{\begin{array}{l}
\mathbf{u}_{d, t}^{j} \\
\mathbf{u}_{d, a_{1}}^{j} \\
\mathbf{u}_{d, a}^{j}
\end{array}\right\}+\left\{\begin{array}{c}
2\left(\mathbf{u}_{d, t}^{j+1}-\mathbf{u}_{d, b}^{j}+\mathbf{u}_{u, t}^{j+1}\right) \\
\mathbf{0} \\
\mathbf{0}
\end{array}\right\}\right),
$$

where $\mathbf{u}_{d, t a}^{j}$ and $\mathbf{u}_{d, t a}^{j}$ are auxiliary variables for the top interface and $\mathbf{K}_{m, n}^{j}$ are the components of approximate half-space stiffness matching with the top of the current slab. Substituting Equations (26) and (27) into Equation (25), we get,

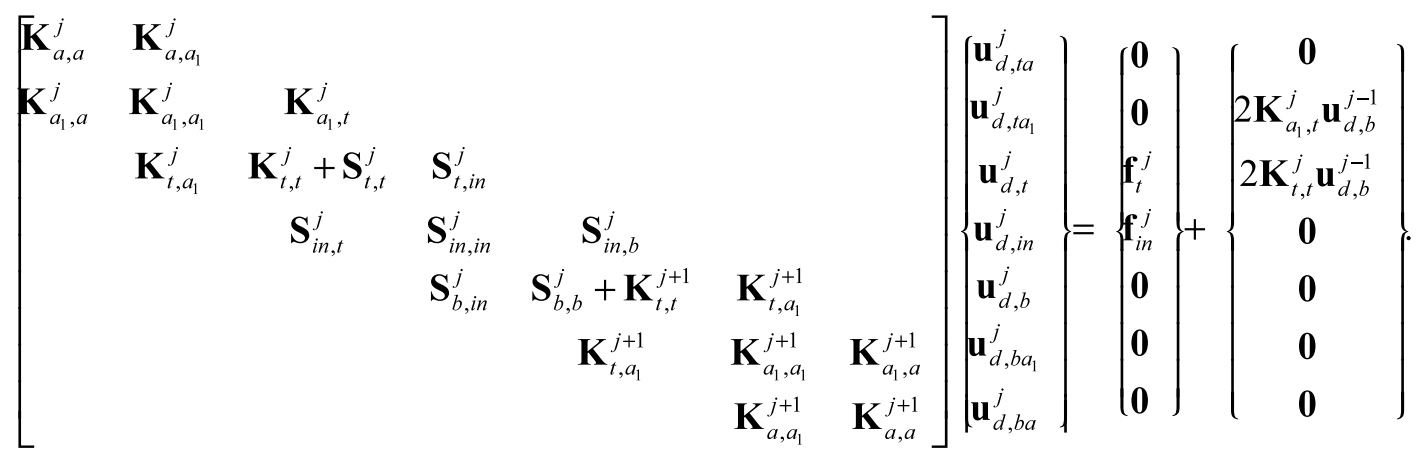

Similar approach can be followed to get the linear system of equations for the upward propagating wavefield:

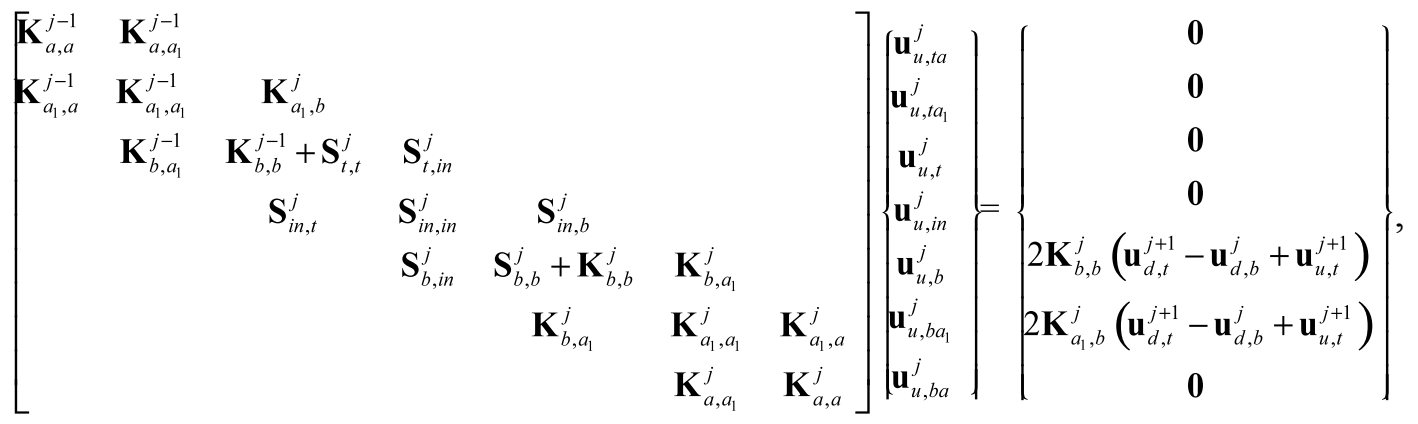

where $\mathbf{u}_{u, t a_{1}}^{j}, \mathbf{u}_{u, t a}^{j}, \mathbf{u}_{u, b a_{1}}^{j}$, and $\mathbf{u}_{u, b a}^{j}$ are auxiliary variables for upward propagation.

Schematics of matrix formulation are shown for downward and upward sweeping in Figure $10(\mathrm{c})$ and $11(\mathrm{c})$, respectively. 
The algorithm is summarized below.

\section{Algorithm 2: Double-Sweeping Solver for 2D/3D Problems}

Step 1: Factorization of slab stiffness

1. for $j=1$ to $n$ (downward propagation)
a. Get interior stiffness in Equation (28): $\mathbf{S}_{m, n}^{j}$
b. Get approximate DtN maps: $\mathbf{K}_{m, n}^{j}$ and $\mathbf{K}_{m, n}^{j+1}$
c. Perform LU factorization of the matrix in Equation (28)

2. for $j=n-1$ to 1 (upward propagators)

a. Get interior stiffness in Equation (29)

b. Get approximate DtN maps: $\mathbf{K}_{m, n}^{j-1}$ and $\mathbf{K}_{m, n}^{j}$

c. Perform LU factorization of the matrix in Equation (29)

Step 2: Determine approximate wavefield

3. for $j=1$ to $n$ (downward wavefield)

a. Compute force vector in Equation (28)

b. Solve the linear system for current slab to get $\mathbf{u}_{d}^{j}$

4. for $j=n-1$ to 1 (upward wavefield)

a. Compute the incident wave at bottom interface $\mathbf{u}_{d, t}^{j+1}-\mathbf{u}_{d, b}^{j}+\mathbf{u}_{u, t}^{j+1}$

b. Compute force vector in Equation (29)

c. Solve the linear system for current slab to get $\mathbf{u}_{u}^{j}$

5. get approximate wave field $\mathbf{u}=\mathbf{u}_{d}+\mathbf{u}_{u}$

\section{Accuracy of the Proposed Method as an approximate Solver}

We test the accuracy of the proposed method for wave propagation in a 2-D medium presented in Figure 12. The domain is discretized using a $200 \times 200$ mesh of dispersion reducing finite elements [24]. The source is a Ricker pulse with central frequency of $20 \mathrm{~Hz}$ positioned at center of the domain. We use 20 horizontal slabs with thickness of 10 elements to get approximate solution, and DtN maps are approximated by 5 PMDL layers. The true (full-wave equation) and approximate solutions are compared for the frequency of $20 \mathrm{~Hz}$ in Figure 14. We also get the time-history of the wavefield by performing the analysis for 
frequency range of $0.4 \mathrm{~Hz}$ to $100 \mathrm{~Hz}$ with the increment of $0.4 \mathrm{~Hz}$, followed by inverse Fourier transform. True and approximate wavefields are compared in Figure 13. These results illustrates that the present method yields a fairly accurate solution for the Helmholtz in the 2-D domain, while expectedly not capturing the multiple reflections.

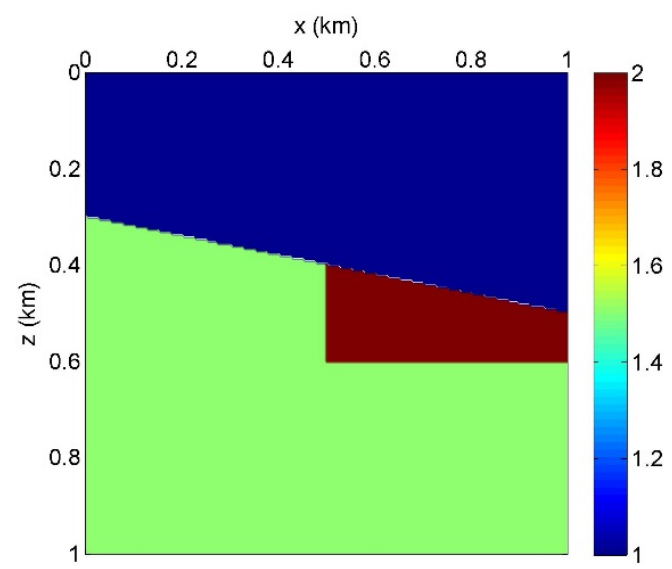

Figure 12. Velocity model for wave propagation simulation.

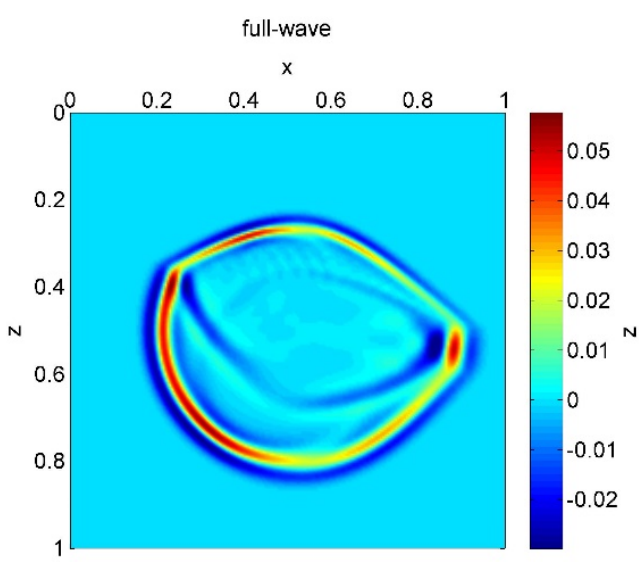

(a)

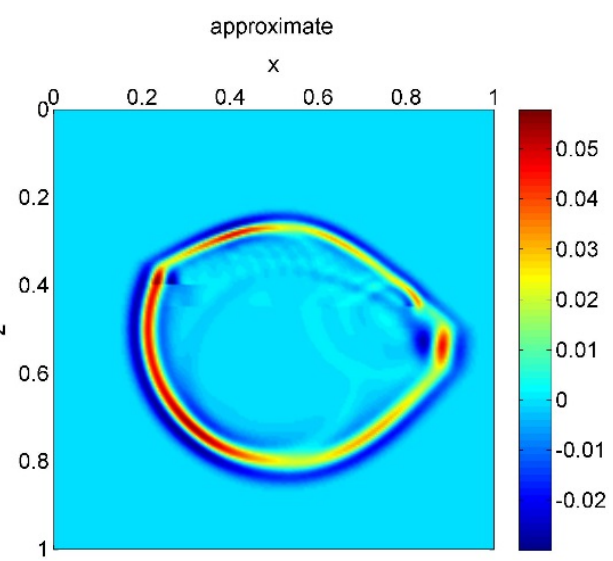

(b)

Figure 13. Comparison of the wavefield at $t=0.245 \mathrm{~s}$. a) true (full-wave) solution. b) Approximate solution. 


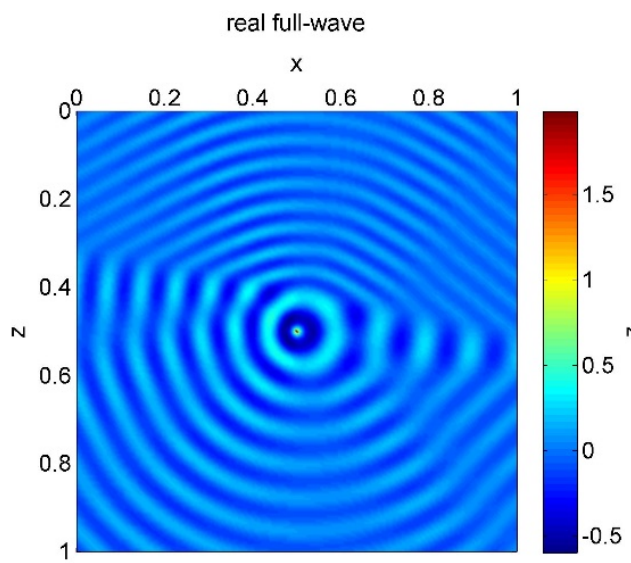

(a)

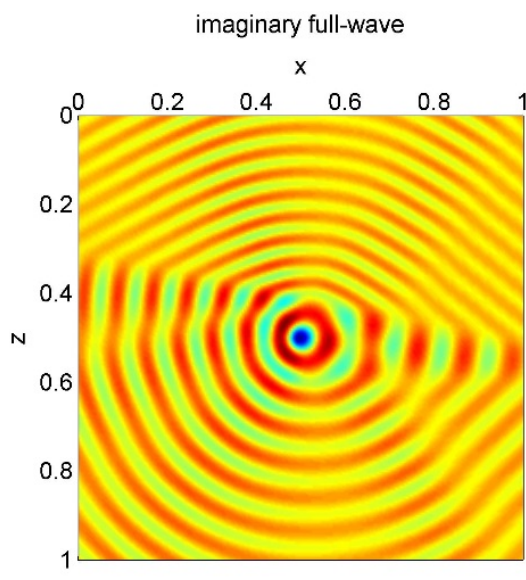

(c)

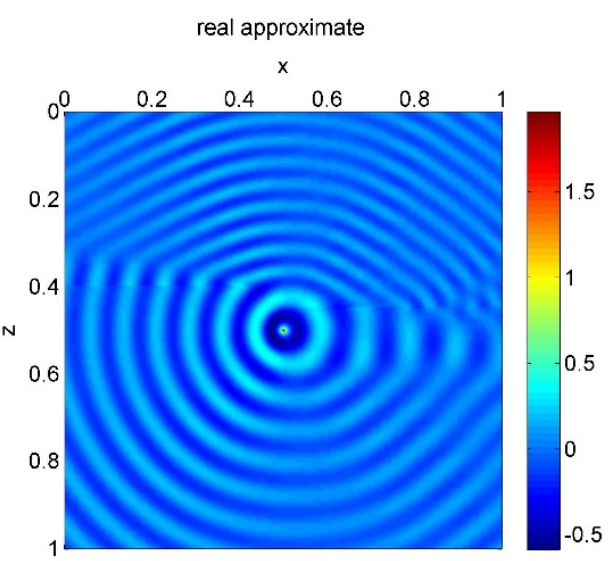

(b)

imaginary approximate

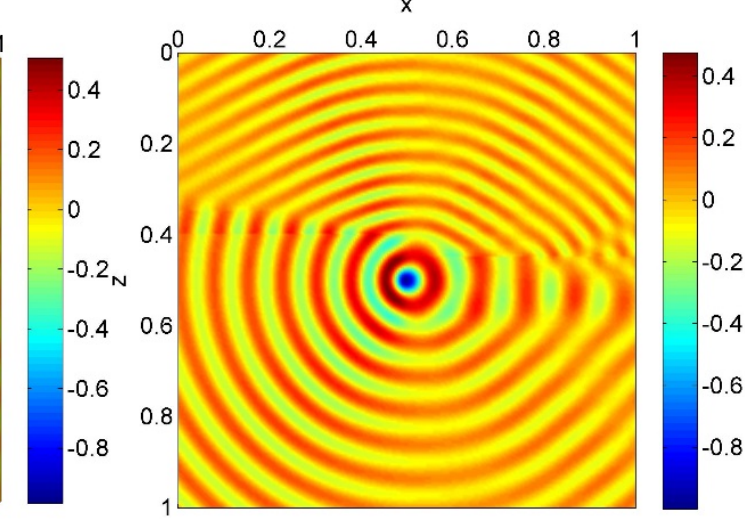

(d)

Figure 14. Comparison of the wavefield at frequency $20 \mathrm{~Hz}$. a,b) real part of the solutions. $\mathrm{c}, \mathrm{d})$ imaginary part of the solution.

\section{Effectiveness of the Proposed Method as a Preconditioner}

We demonstrate the performance of the proposed method as a preconditioner (Algorithm 2) in various 2-D problems, and compare with the sweeping preconditioner. The method has been implemented in MATLAB, and all the tests are carried out on a quad-core $2.83 \mathrm{GHz}$ computer with 64 GB RAM. 


\subsection{Wave Propagation in Unbounded Domain}

In this test, we consider wave propagation in unbounded domains with two different velocity variations:

1) Random velocity, uniformly distributed over the interval ranging from $(0.7,1.3)$ (Figure 15-a).

2) A homogenous domain with $c=0.75$ with a circular inclusion of radius 0.1 , centered at $(0.75,0.75)$, with $c=1.25$ (Figure 15-b).

Two different excitations are considered:

1) Point source located at the center of the domain.

2) Gaussian wave packet borrowed from [11]

$$
f(x, z)=\exp \left(-4 \omega\left(\left(x-x_{0}\right)^{2}+\left(z-z_{0}\right)^{2}\right)\right) \exp \left(i \omega\left(x d_{x}+z d_{z}\right)\right),
$$

where $x_{0}=z_{0}=1 / 8$ and $d_{x}=d_{z}=1 / \sqrt{2}$.

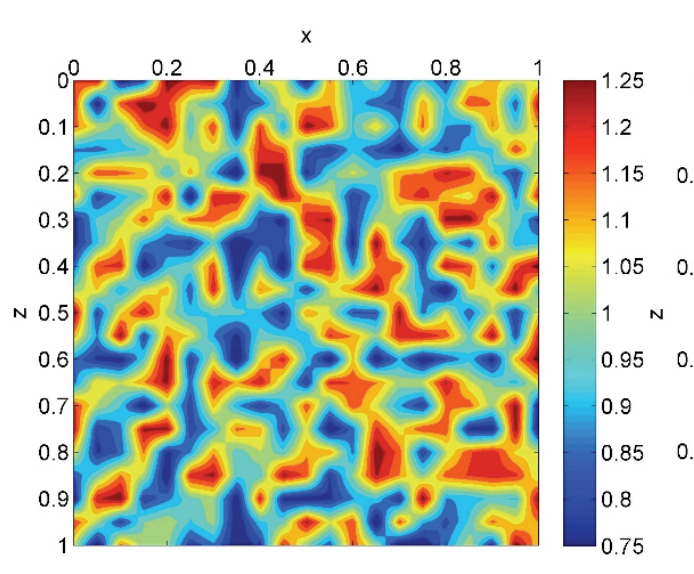

(a)

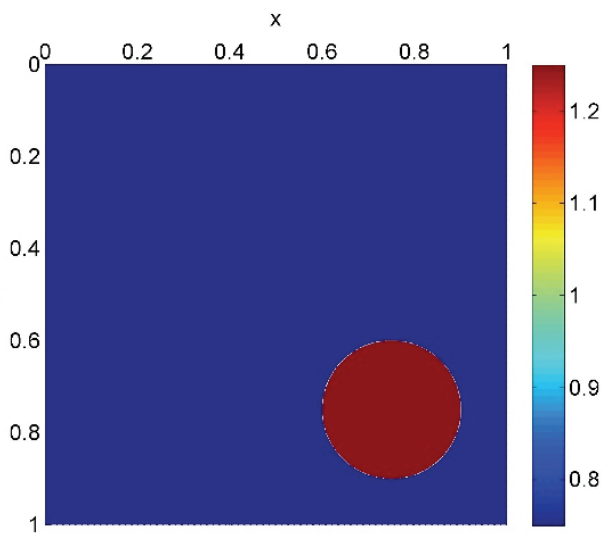

(b)

Figure 15 . Velocity model for numerical tests. a) random medium and b) domain with inclusion.

\subsubsection{Example 1: Frequency Dependence}

For this example, we target a relative error of 1 e- 6 in the residual, and carry out the simulation for multiple frequencies from $8 \mathrm{~Hz}$ to $256 \mathrm{~Hz}$. The finite element discretization is 
chosen such that there are at least 8 elements per wavelength. The horizontal slabs are taken to be 12 elements thick, and the interface conditions are approximated using 5 PMDL layers with equal complex lengths $(2+2 i) /(2 \pi f)$ where $f$ is the cyclic frequency. Table 2 and Table 3 show the number of iterations required to reach the target error. We also report the time required to factorize the preconditioner (Step 1 in Algorithm 5.2) and time needed for GMRES solver in these tables. The wavefields are plotted in Figure 16 and 17 for the frequency of $32 \mathrm{~Hz}$. Based on these results, we make the following observations:

- The number of iterations is almost independent from frequency and mesh size, which is a highly desirable quality of a preconditioner for Helmholtz Equation [6].

- The required time to factorize the preconditioner increases by factor of 4 when the frequency and mesh size are doubled. This observation is consistent with Step 1 in Algorithm 5.2 where standard LU factorization for sparse matrices is used. This property is also reported in [11] for sweeping preconditioner.

Table 2. Results for unbounded domain with random velocity

\begin{tabular}{ccccccc}
\hline \multirow{2}{*}{$\begin{array}{c}\text { Frequency } \\
(\mathrm{Hz})\end{array}$} & Mesh Size & $T^{*}{ }_{\text {setup }}$ & & \multicolumn{2}{c}{ Unit load } & \multicolumn{2}{c}{ Gaussian Wave Packet } \\
\cline { 5 - 7 } & & & & & & \\
\end{tabular}




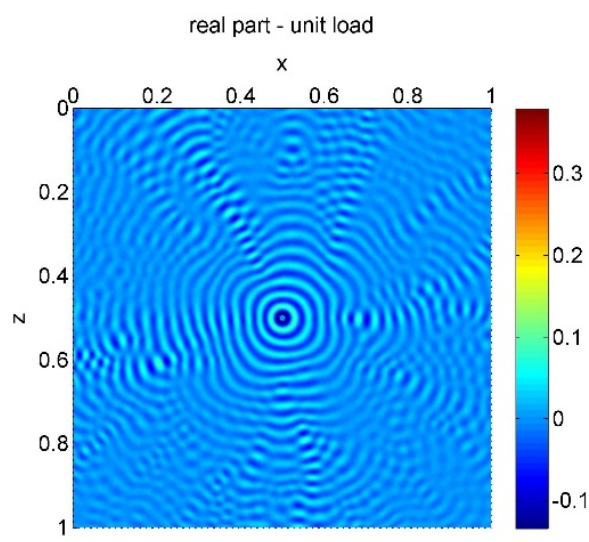

(a)

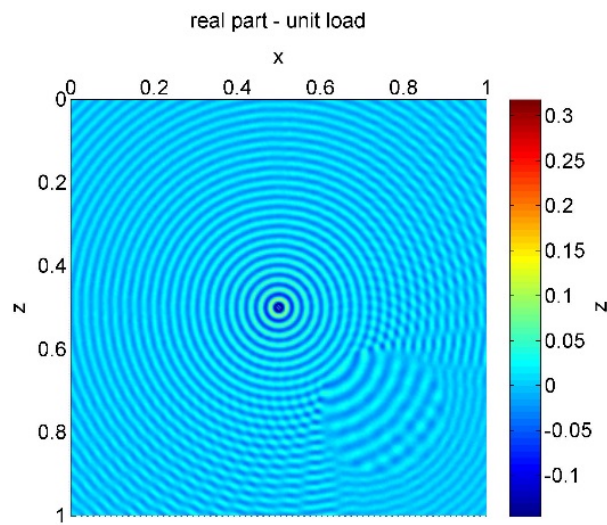

(c)

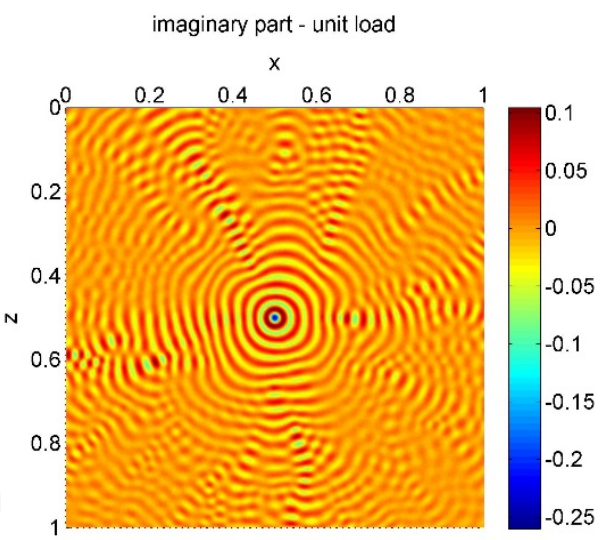

(b)

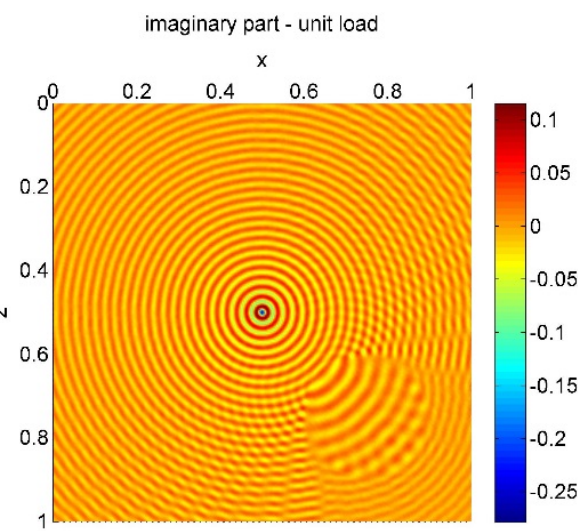

(d)

Figure 16. Solution for a unit load at frequency $32 \mathrm{~Hz}$. a,b) random velocity, c,d) inclusion.

Table 3. Results for unbounded homogenous domain with inclusion

\begin{tabular}{ccccccc}
\hline \multirow{2}{*}{$\begin{array}{c}\text { Frequency } \\
(\mathrm{Hz})\end{array}$} & Mesh Size & $T_{\text {setup }}$ & Iteration & $T_{\text {solve }}$ & Iteration & $T_{\text {solve }}$ \\
\cline { 5 - 7 } & & & & & & \\
\hline 8 & $64 \times 64$ & 0.67 & 4 & 0.17 & 4 & 0.14 \\
16 & $128 \times 128$ & 1.08 & 4 & 0.24 & 4 & 0.29 \\
32 & $256 \times 246$ & 3.78 & 5 & 1.04 & 5 & 1.07 \\
64 & $512 \times 512$ & 14.33 & 5 & 4.23 & 5 & 4.62 \\
128 & $256 \times 256$ & 60.00 & 5 & 17.25 & 5 & 19.02 \\
256 & $2048 \times 2048$ & 242.68 & 6 & 87.85 & 5 & 76.35 \\
\hline
\end{tabular}




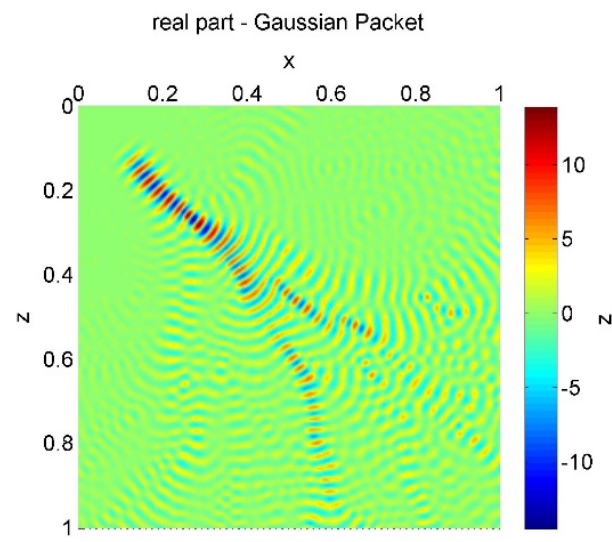

(a)

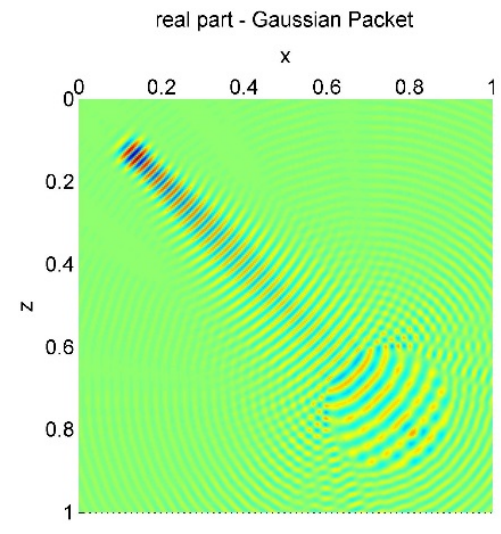

(c)

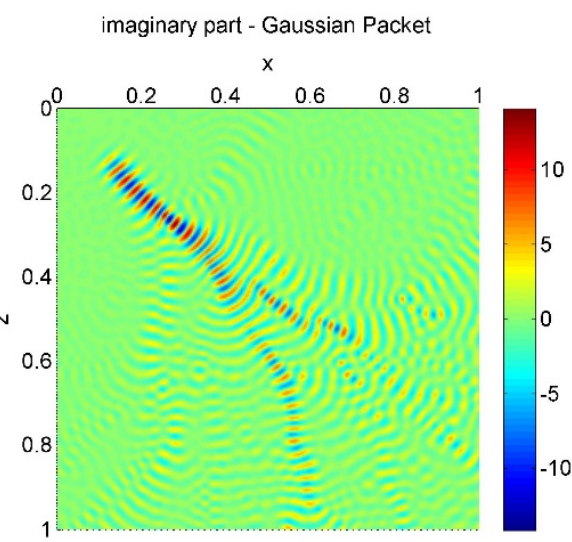

(b)

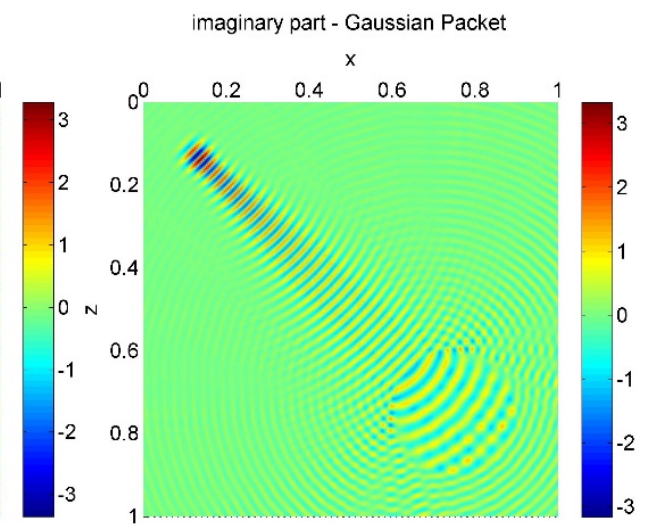

(d)

Figure 17. Solution for a Gaussian packet at frequency $32 \mathrm{~Hz}$. a,b) random velocity, c,d) inclusion.

\subsubsection{Example 2: Effect of Element Size for Fixed Frequency}

In this example, we investigate the effect of element size on the convergence with the proposed preconditioner. We analyze similar problems described in previous section for a single frequency of $32 \mathrm{~Hz}$, but with $8,16,32$, and 64 elements per average wavelength. The horizontal slabs are 12-element thick. The results are presented in Table 4 and 
Table 5, which indicate that the number of iterations is almost independent of mesh size. They also indicate that increasing number of slabs (subdomains) has negligible effect on convergence rate.

Table 4. Results for unbounded random velocity domain in single frequency $32 \mathrm{~Hz}$

\begin{tabular}{|c|c|c|c|c|c|c|c|}
\hline \multirow{2}{*}{$\begin{array}{l}\text { Number of } \\
\text { Elements per } \\
\text { Wavelength }\end{array}$} & \multirow[b]{2}{*}{ Mesh Size } & \multirow{2}{*}{$\begin{array}{l}\text { Number } \\
\text { of Slabs }\end{array}$} & \multirow{2}{*}{$\begin{array}{c}T_{\text {setup }} \\
\text { (s) }\end{array}$} & \multicolumn{2}{|c|}{ Unit load } & \multicolumn{2}{|c|}{ Gaussian Wave Packet } \\
\hline & & & & Iteration & $\begin{array}{c}T_{\text {solve }} \\
\text { (s) }\end{array}$ & Iteration & $T_{\text {solve }}(\mathrm{s})$ \\
\hline 8 & $256 \times 256$ & 22 & 3.74 & 6 & 1.40 & 6 & 1.43 \\
\hline 16 & $512 \times 512$ & 43 & 15.41 & 6 & 6.19 & 6 & 6.11 \\
\hline 32 & $1024 \times 1024$ & 86 & 60.85 & 6 & 24.80 & 6 & 24.24 \\
\hline 64 & $2048 \times 2048$ & 171 & 243.21 & 6 & 101.11 & 6 & 101.45 \\
\hline
\end{tabular}

Table 5. Results for unbounded homogenous domain with inclusion at single frequency 32 $\mathrm{Hz}$

\begin{tabular}{|c|c|c|c|c|c|c|c|}
\hline \multirow{2}{*}{$\begin{array}{l}\text { Number of } \\
\text { Elements per } \\
\text { Wavelength }\end{array}$} & \multirow[b]{2}{*}{ Mesh Size } & \multirow{2}{*}{$\begin{array}{l}\text { Number } \\
\text { of Slabs }\end{array}$} & \multirow{2}{*}{$\begin{array}{c}T_{\text {setup }} \\
\text { (s) }\end{array}$} & \multicolumn{2}{|c|}{ Unit load } & \multicolumn{2}{|c|}{ Gaussian Wave Packet } \\
\hline & & & & Iteration & $\begin{array}{c}T_{\text {solve }} \\
\text { (s) }\end{array}$ & Iteration & $T_{\text {solve }}(\mathrm{s})$ \\
\hline 8 & $256 \times 256$ & 22 & 3.78 & 5 & 1.04 & 5 & 1.14 \\
\hline 16 & $512 \times 512$ & 43 & 15.00 & 5 & 4.60 & 5 & 4.71 \\
\hline 32 & $1024 \times 1024$ & 86 & 59.95 & 5 & 18.96 & 5 & 21.14 \\
\hline 64 & $2048 \times 2048$ & 171 & 244.67 & 5 & 86.83 & 5 & 89.26 \\
\hline
\end{tabular}

\subsubsection{Example 3: Effect of Midpoint Integration and Number of PMDL layers}

To illustrate the benefit of midpoint integration, we compare the number of GMRES iterations with PMDL that uses midpoint integration, as PML-like layers, i.e. without the use of midpoint integration. We also examine the effect of number of the absorbing layers in the effectiveness of preconditioner by using 1, 3, 5, 10 and 20 layers for each subdomain. The simulations are performed for random velocity medium for a single frequency of $32 \mathrm{~Hz}$ and 8 elements per average wave length. Each subdomain consists of 12 finite element layers. The results are summarized in Table 6 and Table 7. As illustrated in these tables, mid-point 
integration of PMDL, i.e. the use of midpoint integration, leads to significant improvement in efficiency by reducing the number of GMRES iterations.

Table 6. Results for unbounded random velocity domain for a single frequency of $32 \mathrm{~Hz}$ with unit source at the center

\begin{tabular}{ccccccc}
\hline $\begin{array}{c}\text { Number of } \\
\text { Absorbing } \\
\text { Layers }\end{array}$ & \multicolumn{3}{c}{ Mid-point Integration } & \multicolumn{3}{c}{ Standard Integration } \\
\cline { 2 - 7 } & $\begin{array}{c}T_{\text {setup }} \\
(\mathrm{s})\end{array}$ & Iteration & $T_{\text {solve }}$ & $T_{\text {setup }}$ & Iteration & $\begin{array}{c}T_{\text {solve }} \\
(\mathrm{s})\end{array}$ \\
\hline 1 & 2.24 & 11 & 1.35 & 2.27 & 23 & 2.80 \\
3 & 3.06 & 6 & 1.13 & 3.02 & 20 & 3.31 \\
5 & 3.74 & 6 & 1.40 & 4.02 & 21 & 4.14 \\
10 & 7.35 & 6 & 2.39 & 7.37 & 21 & 6.85 \\
20 & 19.07 & 6 & 5.28 & 19.30 & 21 & 15.60 \\
\hline
\end{tabular}

Table 7. Results for unbounded random velocity domain for a single frequency $32 \mathrm{~Hz}$ with Gaussian Wave Packet source

\begin{tabular}{ccccccc}
\hline $\begin{array}{c}\text { Number of } \\
\text { Absorbing } \\
\text { Layers }\end{array}$ & \multicolumn{3}{c}{ Mid-point Integration } & \multicolumn{3}{c}{ Standard Integration } \\
\cline { 2 - 7 } & $\begin{array}{c}T_{\text {setup }} \\
(\mathrm{s})\end{array}$ & Iteration & $\begin{array}{c}T_{\text {solve }} \\
(\mathrm{s})\end{array}$ & $\begin{array}{c}T_{\text {setup }} \\
(\mathrm{s})\end{array}$ & Iteration & $\begin{array}{c}T_{\text {solve }} \\
(\mathrm{s})\end{array}$ \\
\hline 1 & 2.24 & 11 & 1.36 & 2.27 & 22 & 2.65 \\
3 & 3.06 & 6 & 1.13 & 3.02 & 20 & 3.31 \\
5 & 3.74 & 6 & 1.43 & 4.02 & 20 & 4.11 \\
10 & 7.35 & 6 & 2.44 & 7.35 & 20 & 6.87 \\
20 & 19.07 & 6 & 5.38 & 19.30 & 21 & 15.75 \\
\hline
\end{tabular}

\subsubsection{Example 4: Effect of Wave Velocity Variation on Convergence Rate}

To examine the performance of the proposed method for varying velocity contrasts, various velocity ranges are chosen in the random velocity model in Figure 15 -a: $(0.9,1.1)$, $(0.8,1.2),(0.7,1.3),(0.6,1.4)$, and $(0.5,1.5)$. We carried out the analysis for the frequency of $32 \mathrm{~Hz}$, with 8 elements per wavelength and 22 subdomains. Table 8 shows the number of GRMES iterations to convergence and run time for various wave velocity ranges. As these results illustrate widening wave velocity range leads to slight increase in number of GMRES 
iterations, which is expected since higher velocity contrast leads to stronger multiples that are ignored in the preconditioner.

Table 8. Results for unbounded random velocity domain for a single frequency $32 \mathrm{~Hz}$

\begin{tabular}{ccccc}
\hline \multirow{2}{*}{$\begin{array}{c}\text { Velocity } \\
\text { Range }\end{array}$} & \multicolumn{2}{c}{ Unit Load } & \multicolumn{2}{c}{ Gaussian Wave Packet } \\
\cline { 2 - 5 } & $\begin{array}{c}T_{\text {solve }} \\
(\mathrm{s})\end{array}$ & Iteration & $\begin{array}{c}T_{\text {solve }} \\
(\mathrm{s})\end{array}$ & Iteration \\
\hline $0.9-1.1$ & 0.95 & 4 & 0.93 & 3 \\
$0.8-1.2$ & 1.14 & 5 & 1.27 & 5 \\
$0.7-1.3$ & 1.47 & 6 & 1.52 & 6 \\
$0.6-1.4$ & 1.71 & 8 & 1.89 & 8 \\
$0.5-1.5$ & 2.27 & 10 & 2.17 & 10 \\
\hline
\end{tabular}

\subsubsection{Example 5: Comparison with Sweeping Preconditioner}

The performance of the present method is compared with the sweeping preconditioner for the random medium. Both methods are implemented in MATLAB and the corresponding codes are optimized to the same level, and all the tests are carried out on a quad-core $2.83 \mathrm{GHz}$ computer with $64 \mathrm{~GB}$ RAM. The external force is a unit load at the center of the domain. The relative error tolerance for GMRES is $1 \mathrm{e}-6$ and 8 elements per average wavelength are used. The thickness of horizontal layers are 12 elements for both the sweeping preconditioner and the proposed method. Other parameters remained unchanged from previous experiments in Section 4. The results are given in Table 9 and 7. The GMRES convergence rates are also plotted and compared for frequencies 16, 64, and $256 \mathrm{~Hz}$ in Figure 18 and 19.

These results clearly show the advantage of the proposed approach compared to the sweeping preconditioner. Figure 18 and 19 show fast convergence of the present method for both excitations. As stated in Table 9 and 7 the present method requires more effort to setup the preconditioner due to the complexity of the approximation. However, the total computational time is much less than sweeping preconditioner - especially for the problems with multiple excitations or relatively high frequencies. 
Table 9. Comparison of cost and performance for different preconditioners for random velocity field

\begin{tabular}{ccccccccccc}
\hline \multirow{2}{*}{$\begin{array}{c}\text { Frequency } \\
(\mathrm{Hz})\end{array}$} & Mesh Size & \multicolumn{4}{c}{ Sweeping Preconditioner } & \multicolumn{5}{c}{ Present method } \\
\cline { 3 - 10 } & & Iteration & $T_{\text {setup }}$ & $T_{\text {solve }}$ & $T_{\text {total }}{ }^{*}$ & Iteration & $T_{\text {setup }}$ & $T_{\text {solve }}$ & $T_{\text {total }}{ }^{*}$ \\
\hline 8 & $64 \times 64$ & 28 & 0.40 & 0.53 & 0.93 & 6 & 0.68 & 0.18 & 0.86 \\
16 & $128 \times 128$ & 29 & 0.36 & 1.31 & 1.67 & 5 & 1.06 & 0.27 & 1.33 \\
32 & $256 \times 246$ & 33 & 1.22 & 5.69 & 6.91 & 6 & 3.74 & 1.40 & 5.14 \\
64 & $512 \times 512$ & 36 & 4.59 & 28.53 & 33.07 & 7 & 14.62 & 6.66 & 21.28 \\
128 & $256 \times 256$ & 40 & 18.11 & 130.37 & 148.48 & 8 & 59.67 & 26.47 & 86.14 \\
256 & $2048 \times 2048$ & 48 & 75.06 & 725.78 & 800.84 & 9 & 246.18 & 141.56 & 387.76 \\
\hline \multicolumn{1}{c}{}
\end{tabular}

Table 10. Comparison of cost and performance for different preconditioners for velocity field with inclusion

\begin{tabular}{ccccccccccc}
\hline \multirow{2}{*}{$\begin{array}{c}\text { Frequency } \\
(\mathrm{Hz})\end{array}$} & Mesh Size & \multicolumn{3}{c}{ Sweeping Preconditioner } & \multicolumn{5}{c}{ Present method } \\
\cline { 3 - 10 } & & Iteration & $T_{\text {setup }}$ & $T_{\text {solve }}$ & $T_{\text {total }}$ & Iteration & $T_{\text {setup }}$ & $T_{\text {solve }}$ & $T_{\text {total }}$ \\
\hline 8 & $64 \times 64$ & 30 & 0.44 & 0.56 & 1.00 & 4 & 0.67 & 0.17 & 0.84 \\
16 & $128 \times 128$ & 31 & 0.36 & 1.39 & 1.75 & 4 & 1.08 & 0.24 & 1.32 \\
32 & $256 \times 246$ & 32 & 1.21 & 5.20 & 6.41 & 5 & 3.78 & 1.04 & 4.82 \\
64 & $512 \times 512$ & 34 & 4.49 & 26.10 & 30.59 & 5 & 14.33 & 4.23 & 18.56 \\
128 & $256 \times 256$ & 41 & 17.92 & 133.69 & 151.61 & 5 & 60.00 & 17.25 & 77.25 \\
256 & $2048 \times 2048$ & 60 & 74.45 & 940.25 & 1014.7 & 6 & 242.68 & 87.85 & 330.53 \\
\hline
\end{tabular}




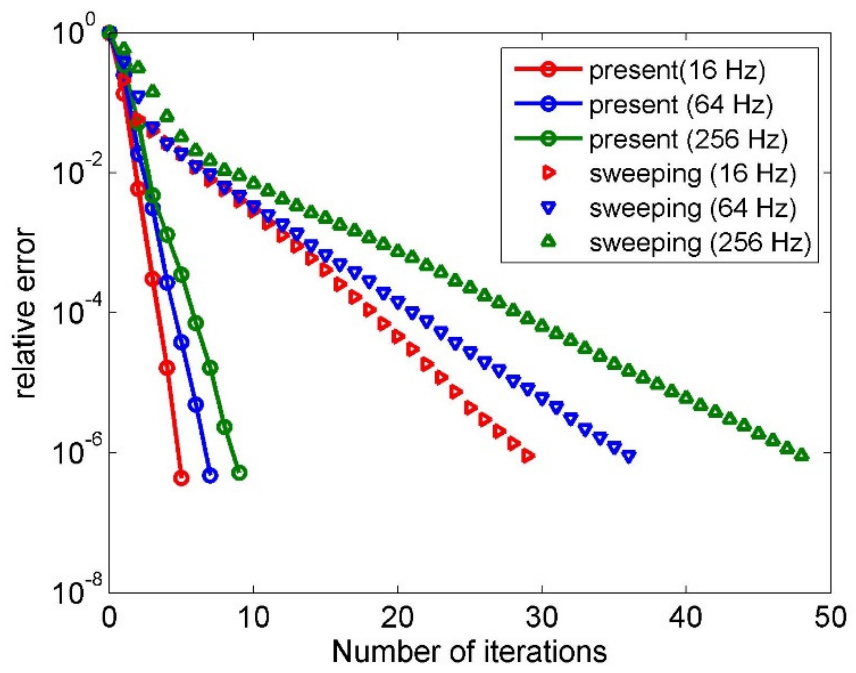

Figure 18. GMRES convergence rate for random velocity field.

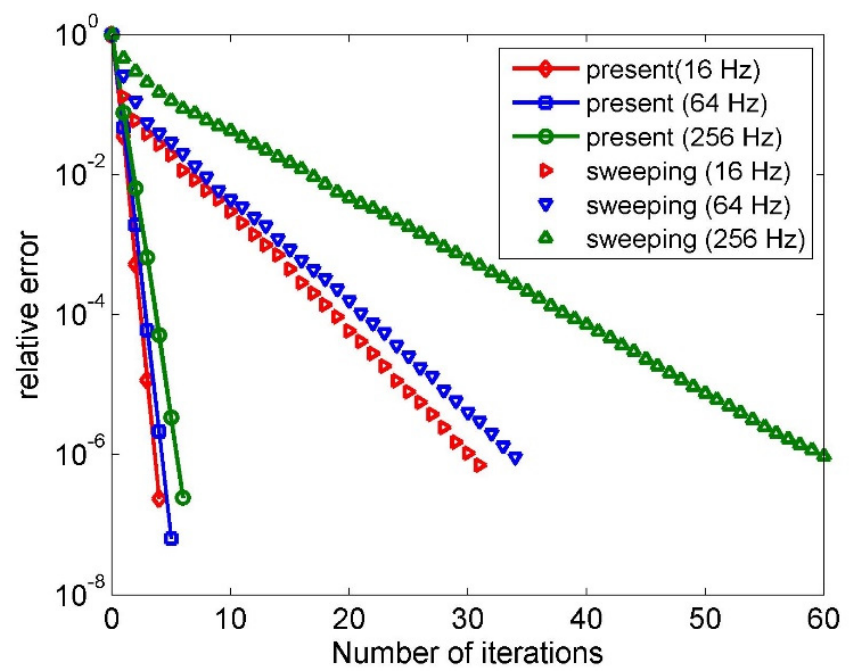

Figure 19. GMRES convergence rate for inclusion velocity.

Similar to the 1-D case, we compare the eigenvalue spectrum of the preconditioned linear systems as another indication of GMRES convergence behavior. Figure 20 compares the eigenvalue amplitudes for the sweeping preconditioner and the proposed method, for two forcing frequencies of 8 and $16 \mathrm{~Hz}$; only the largest and smallest 20 eigenvalues are shown. The eigenvalues are rather dispersed for the sweeping preconditioner, while they are 
clustered for the proposed method, explaining the faster convergence of the proposed method.

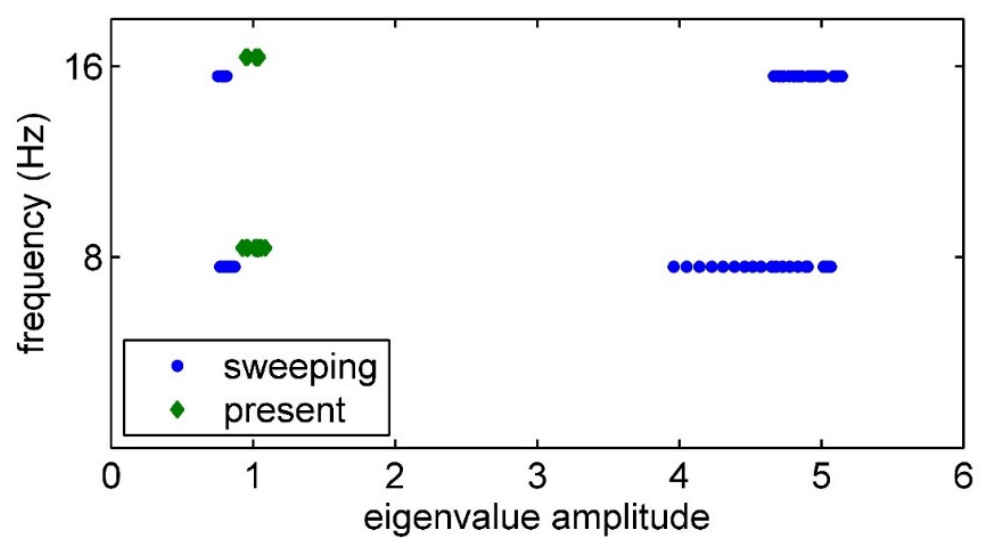

Figure 20. Comparison of eigenvalue spectrum of preconditioned system $\mathbf{P}^{-1} \mathbf{S}$ for sweeping preconditioner and the present method for frequencies 8 and $16 \mathrm{~Hz}$.

\subsection{Bounded Domains with Different Boundary Conditions}

The proposed preconditioner can be used for any configuration of boundary conditions. In this section we study two typical problems: half-space with Neumann boundary condition and a wave guide.

\subsubsection{Example 1: Half-Space with Neumann Boundary Condition}

In this example we consider a unit box with traction free boundary at $z=0$. Absorbing boundary conditions are applied on the other three sides. We consider both random wave velocity and homogenous media with circular inclusion, used in first example. The excitation is applied at the center of the surface, $(x=0.5, z=0)$. Other parameters are the same as previous examples in Section 4. The convergence and computational cost are presented in Table 11 and solutions are given in Figure 21. Similar to the previous experiments with full space domains, the proposed method appears to work well for halfspace problems. 
Table 11. Comparison of cost and performance for half-space problem

\begin{tabular}{cccccccccc}
\hline \multirow{2}{*}{$\begin{array}{c}\text { Frequency } \\
(\mathrm{Hz})\end{array}$} & Mesh Size & \multicolumn{4}{c}{ Random Velocity } & \multicolumn{5}{c}{ Homogenous with inclusion } \\
\cline { 3 - 10 } & & Iteration & $T_{\text {setup }}$ & $T_{\text {solve }}$ & $T_{\text {total }}$ & Iteration & $T_{\text {setup }}$ & $T_{\text {solve }}$ & $T_{\text {total }}$ \\
\hline 8 & $64 \times 64$ & 7 & 0.63 & 0.21 & 0.84 & 5 & 0.72 & 0.17 & 0.89 \\
16 & $128 \times 128$ & 6 & 1.06 & 0.38 & 1.44 & 5 & 1.05 & 0.28 & 1.33 \\
32 & $256 \times 246$ & 7 & 3.74 & 1.47 & 5.21 & 5 & 3.71 & 1.24 & 4.95 \\
64 & $512 \times 512$ & 7 & 14.79 & 6.10 & 20.89 & 5 & 14.64 & 5.18 & 18.82 \\
128 & $256 \times 256$ & 8 & 60.48 & 31.64 & 92.12 & 6 & 60.60 & 21.95 & 92.95 \\
256 & $2048 \times 2048$ & 8 & 245.08 & 128.43 & 373.51 & 7 & 244.23 & 103.37 & 347.60 \\
\hline
\end{tabular}

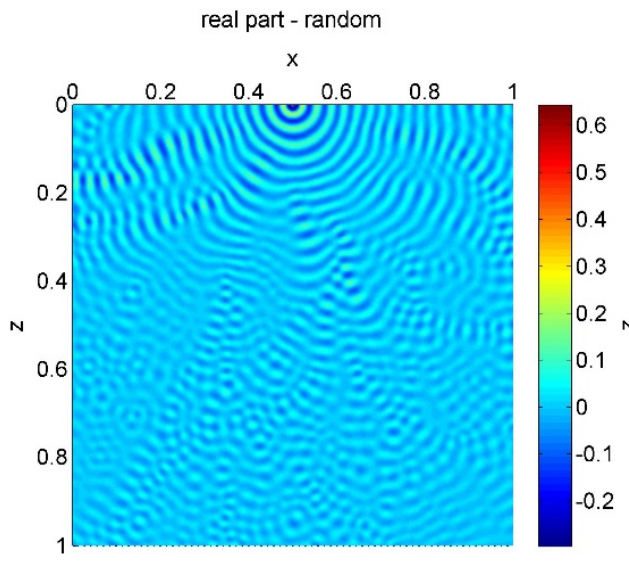

(a)

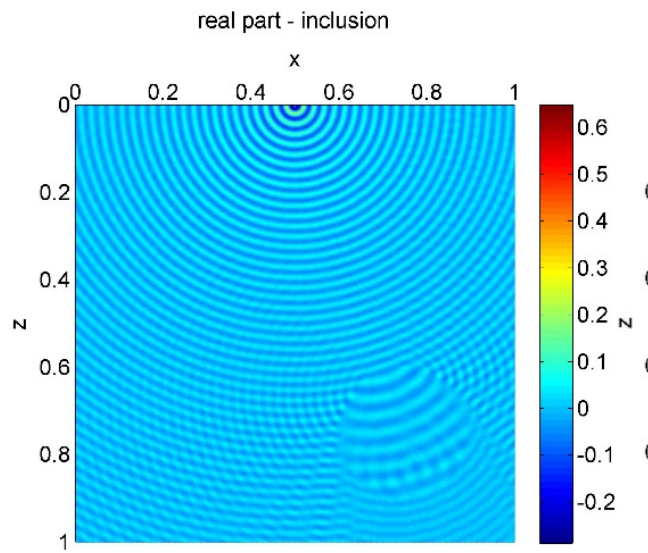

(c)

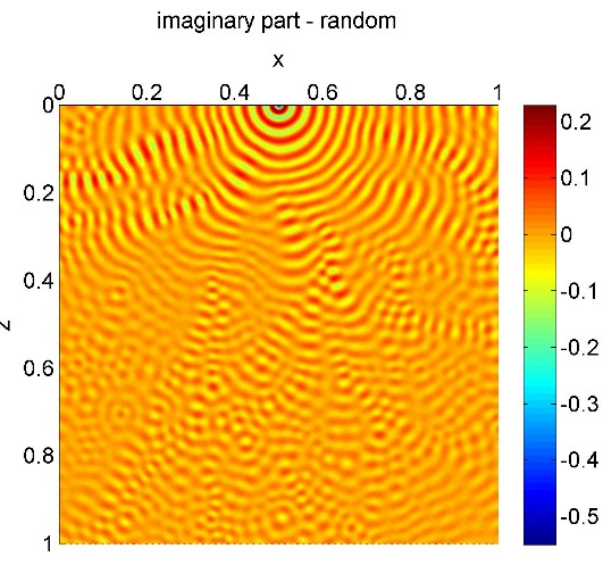

(b)

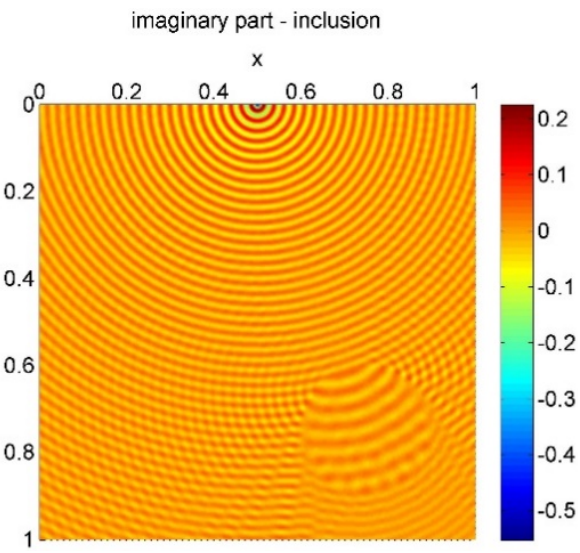

(d)

Figure 21. Solution for half-space problem at $32 \mathrm{~Hz}$. a,b) real and imaginary parts for random velocity. c,d) real and imaginary part for the inclusion model. 


\subsubsection{Example 2: wave guide problem}

We assume a homogenous wave guide in the $z$ direction with $\mathrm{c}=1.0 \mathrm{~m} / \mathrm{s}$, fixed on left and right boundaries at $x=0$ and $x=1$. A unit excitation is located at $x=0.5$ and $z=0$. We try two different ways to partition the domain. In the first case, we divide the domain into horizontal layers and apply preconditioner that consists are downward sweeping followed by upward sweeping. In the second case, the domain is sliced into vertical layers and the marching is performed horizontally from left to right, then right to left. 8 elements per wavelength are used, and the layers are 12-elements thick for both cases. Other parameters are similar to the examples in Section 4. The number of iterations and cost are compared in Table 12 and Figure 23. As seen from the table, the direction of marching has significant effect on convergence rate and thus the cost of the solution. This is not surprising because the standing waves between the parallel boundaries of the waveguide are essentially results of infinite reflections, and the double sweeping is not a good approximation of the final solution, leading to poor convergence. On the other hand, when the sweeping is done in the direction of unboundedness, there are no standing waves and the convergence is much better. The implication of this observation is that whenever feasible it is best to perform double sweeping in the direction of unboundedness.

Table 12. Comparison of cost and performance for wave guide problem

\begin{tabular}{cccccccccc}
\hline \multirow{2}{*}{$\begin{array}{c}\text { Frequency } \\
(\mathrm{Hz})\end{array}$} & Mesh Size & \multicolumn{4}{c}{ Horizontal Blocks } & \multicolumn{5}{c}{ Vertical Blocks } \\
\cline { 3 - 10 } & & Iteration & $T_{\text {setup }}$ & $T_{\text {solve }}$ & $T_{\text {total }}$ & Iteration & $T_{\text {setup }}$ & $T_{\text {solve }}$ & $T_{\text {total }}$ \\
\hline 8 & $64 \times 64$ & 3 & 0.53 & 0.16 & 0.69 & 11 & 0.69 & 0.27 & 0.96 \\
16 & $128 \times 128$ & 3 & 0.88 & 0.19 & 1.07 & 15 & 1.04 & 0.78 & 1.82 \\
32 & $256 \times 246$ & 3 & 3.38 & 0.88 & 4.26 & 21 & 3.74 & 4.41 & 8.15 \\
64 & $512 \times 512$ & 6 & 13.54 & 5.39 & 18.93 & 31 & 14.75 & 30.21 & 44.96 \\
128 & $256 \times 256$ & 10 & 56.49 & 39.70 & 96.19 & 45 & 60.87 & 198.15 & 259.02 \\
256 & $2048 \times 2048$ & 23 & 231.68 & 371.31 & 602.99 & 58 & 248.34 & 1101 & 1349 \\
\hline
\end{tabular}




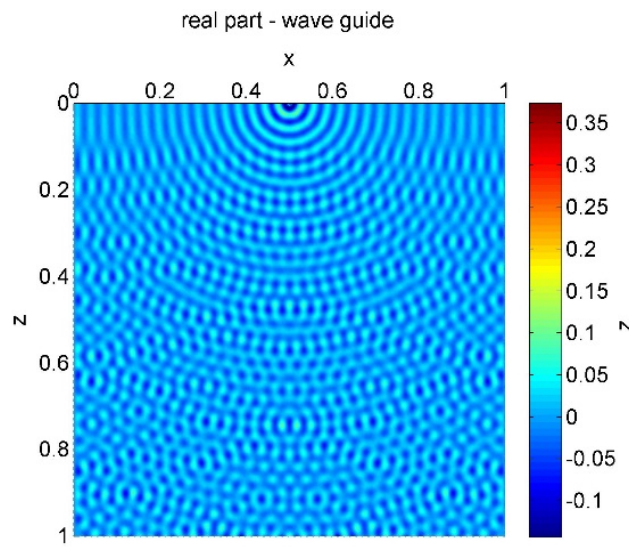

(a)

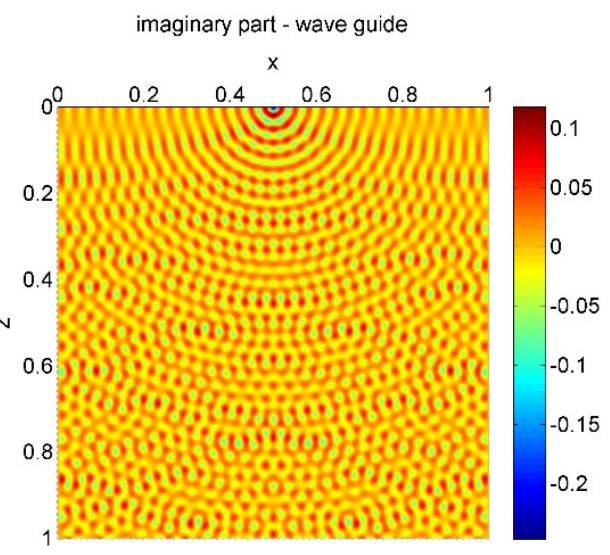

(b)

Figure 22. Solution for wave guide at $32 \mathrm{~Hz}$. a) real part and b) imaginary part.

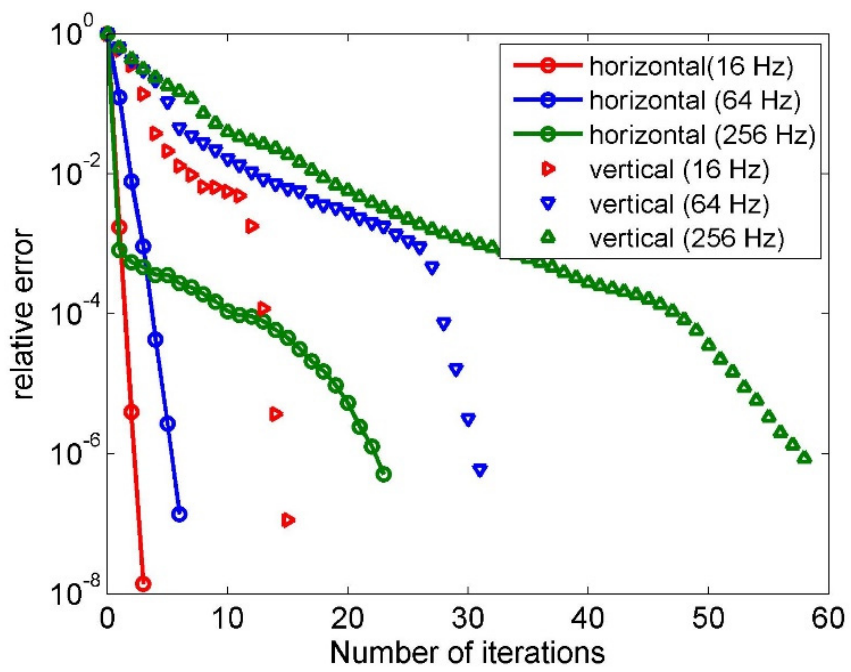

Figure 23. Comparison of GMRES convergence rate for wave guide problem. 


\section{Summary}

We developed a new preconditioner for the Helmholtz equation in heterogeneous media. The preconditioner is essentially an approximate solver for the wave equation which ignores the effect of multiple reflections. The approach involves two main ideas: (a) partitioning the solution into down going wave considering only primary transmissions, and upcoming wave considering only primary reflections, and (b) sequentially solving for down going and then upcoming wavefield, facilitated by special interface conditions that explicitly neglect the multiple reflections. It is shown that such an approach preserves the amplitude information for the large part (except for the multiples), and hence named amplitudepreserving propagator. This approximate solver, when viewed as a preconditioner, is a good approximation of the inverse of the wave operator, at the same time, highly efficient due to the sequential solution procedure. Indeed, the preconditioner is shown to be effective with the help of extensive numerical examples, both in 1-D and 2-D. Specifically, we observe three significant properties of the proposed preconditioner. First, number of iterations grows very slowly with increasing frequency. Second, the discretization size and number of subdomains do not change the number of iterations. Third, and most importantly, the computational time is significantly less than a similar preconditioner, namely the sweeping preconditioner.

\section{Acknowledgment}

This material is based upon work supported by the National Science Foundation under Grant No. DMS-1016514. The authors wish to thank Dr. Senganal Thirunavukkarasu for many helpful discussions, and Ali Vaziri for his thorough review. 


\section{References}

[1] M. J. Gander, Schwarz methods over the course of time, Electron. Trans. Numer. Anal. 31 (2008) 228-255.

[2] A. Vion and C. Geuzaine, Double sweep preconditioner for optimized Schwarz methods applied to the Helmholtz problem, J. Comput. Phys. 266, (2014) 171-190.

[3] M. Gander and G. Wanner, From Euler, Ritz, and Galerkin to modern computing, SIAM Rev. 54 (2012) 627-666.

[4] O. G. Ernst and M. J. Gander, Why it is difficult to solve Helmholtz problems with classical iterative methods, in: I. G. Graham, T. Y. Hou, O. Lakkis, and R. Scheichl, (Eds.), Numerical Analysis of Multiscale Problems, Springer Berlin Heidelberg, 2012, pp. 325-363.

[5] J. Poulson, B. Engquist, S. Li, and L. Ying. A parallel sweeping preconditioner for heterogeneous 3D Helmholtz equations. SIAM Journal on Scientific Computing 35, no. 3 (January 1, 2013): C194-212.

[6] Y. A. Erlangga, Advances in iterative methods and preconditioners for the Helmholtz equation, Arch. Comput. Methods Eng. 15 (2008) 37-66.

[7] Y. Saad, Iterative Methods for Sparse Linear Systems, Second ed., Society for Industrial and Applied Mathematics, Philadelphia, 2003.

[8] M. J. Gander and F. Nataf, An incomplete LU preconditioner for problems in acoustics, J. Comput. Acoust. 13 (2005) 455-476.

[9] A. Bayliss, C. I. Goldstein, and E. Turkel, An iterative method for the Helmholtz equation, J. Comput. Phys. 49 (1983) 443-457.

[10] Y. A. Erlangga, C. Vuik, and C. W. Oosterlee, On a class of preconditioners for solving the Helmholtz equation, Appl. Numer. Math. 50 (2004) 409-425.

[11] B. Engquist and L. Ying, Sweeping preconditioner for the Helmholtz equation: moving perfectly matched layers, Multiscale Model. Simul. 9 (2011) 686-710.

[12] C. C. Stolk, A rapidly converging domain decomposition method for the Helmholtz equation, J. Comput. Phys. 241 (2013) 240-252. 
[13] M. Eslaminia and M. Guddati, A novel wave equation solver to increase the efficiency of full waveform inversion, in: SEG Technical Program Expanded Abstracts 2014, Society of Exploration Geophysicists, 2014, pp. 1028-1032.

[14] Eslaminia, M., Amplitude-Preserving Propagator and Its Applications in Computational Wave Propagation and Seismic Imaging, PhD dissertation, North Carolina State University, Raleigh, NC, 2014.

[15] D. Colton and R. Kress, Inverse Acoustic Obstacle Scattering, in: Inverse Acoustic and Electromagnetic Scattering Theory, Springer New York, 2013, pp. 119-186.

[16] M. N. Guddati and K.-W. Lim, Continued fraction absorbing boundary conditions for convex polygonal domains, Int. J. Numer. Methods Eng. 66 (2006) 949-977.

[17] E. L. Lindman, 'Free-space' boundary conditions for the time dependent wave equation,” J. Comput. Phys. 18 (1975) pp. 66-78.

[18] B. Engquist and A. Majda, Radiation boundary conditions for acoustic and elastic wave calculations, N. Y. Univ. Conf. Sci. Comput. N. Y. N Apr 20-22 1977, Commun. Pure Appl. Math. 32 (1979) 313-357.

[19] R. L. Higdon, Absorbing boundary conditions for difference approximations to the multidimensional wave equation, Math. Comput. 47 (1986) 437-459.

[20] J.-P. Berenger, A perfectly matched layer for the absorption of electromagnetic waves, J. Comput. Phys. 114 (1994) 185-200.

[21] W. C. Chew and W. H. Weedon, A 3D perfectly matched medium from modified Maxwell's equations with stretched coordinates, Microw. Opt. Technol. Lett., 7 (1994) 599-604.

[22] M. N. Guddati and J. L. Tassoulas, Continued-fraction absorbing boundary conditions for the wave equation, J. Comput. Acoust. 8 (2000) 139-156.

[23] M. N. Guddati, K. W. Lim, and M. A. Zahid, Perfectly matched discrete layers for unbounded domain modeling, in: F. Magoulès, (Ed.) Computational Science, Engineering \& Technology Series, vol. 18, Stirlingshire, UK: Saxe-Coburg Publications, 2008, pp. 69-98.

[24] M. N. Guddati and B. Yue, Modified integration rules for reducing dispersion error in finite element methods, Comput. Methods Appl. Mech. Eng. 193 (2004) 275-287. 
[25] D. Givoli, High-order local non-reflecting boundary conditions: a review, Wave Motion, 39 (2004) 319-326, Apr. 2004.

[26] S. Asvadurov, V. Druskin, M. N. Guddati, and L. Knizhnerman, On optimal finitedifference approximation of PML, SIAM J. Numer. Anal. 41 (2004) 287-305.

[27] S. Savadatti, and M. N. Guddati. A finite element alternative to infinite elements, Computer Methods in Applied Mechanics and Engineering 199, no. 33-36 (July 1, 2010): 2204-23. 


\section{Appendix A: Approximate Dirichlet to Neumann Map}

Since the exact DtN map or half-space stiffness is a non-local operator in higher dimensions and computationally impractical, it is approximated using local absorbing boundary conditions (ABCs). Numerous methods have been proposed for this purpose over past few decades and a brief summary of them can be found in [25]. Local ABCs are often classified into two categories. First class approximates the DtN map using rational functions, sometimes referred to as rational ABCs [17-19]. Second group of methods is called perfectly matched layer (PML), which approximate wave absorption into half-space with the help of truncated attenuating medium with matching impedance [20,21]. In the past decade, these two methods are shown to have links [26], leading to the development of perfectly matched discrete layer (PMDL) [22, 23], which is essentially a simple and effective discretization of PML, but is equivalent to rational approximation of the DtN map. Thus, PMDL inherits the flexibility of PML with respect to extension to more complex media, and the efficiency of rational absorbers [16]. Because of this advantage, we use PMDL in the current study. We however note that other approaches of standard PML or rational ABC can also be used easily in the proposed framework.

We consider following wave equation,

$$
-\frac{\partial^{2} u}{\partial x^{2}}-\frac{\partial^{2} u}{\partial z^{2}}-\frac{\omega^{2}}{c^{2}} u=0,
$$

where $\omega$ is temporal frequency and $c$ is wave velocity. To simplify the formulation, we take Fourier transform of Equation (A-1) in $x$ direction to get,

$$
-\frac{\partial^{2} u}{\partial z^{2}}-k^{2} u=0,
$$

where we use the duality $\frac{\partial}{\partial x} \leftrightarrow i k_{x}$, and $k=\sqrt{\omega^{2} / c^{2}-k_{x}^{2}}$. The solution takes the form $A e^{i k z}+B e^{-i k z}$. If we consider the half-space, $\left(z_{0}, \infty\right)$, the radiation condition indicates that the waves should be downward propagating (in the positive $\mathrm{z}$ direction), indicating that the 
wavenumber should be positive. Thus, the solution takes the form: $A e^{i k z}$. With this solution form, we immediately have a relation between traction and wavefield at the boundary $z=z_{0}$ :

$$
-\left.\frac{\partial u}{\partial z}\right|_{z=z_{0}}=-i k u_{0},
$$

where $u_{0}$ is the wavefield amplitude at $z=z_{0}$. Thus the half-space stiffness is essentially:

$$
\lambda=-i k=-i \sqrt{\omega^{2} / c^{2}-k_{x}^{2}} .
$$

While the above expression is quite simple in Fourier domain, inverse Fourier transforming in $x$ renders the operator nonlocal and expensive. PMDL essentially is a mesh extension technique that can be applied to Equations (A-1) and (A-2) alike, resulting in accurate approximation of the DtN map. We first summarize the idea of PMDL for Equation (A-2) and comment on extension to Equation (A-1).
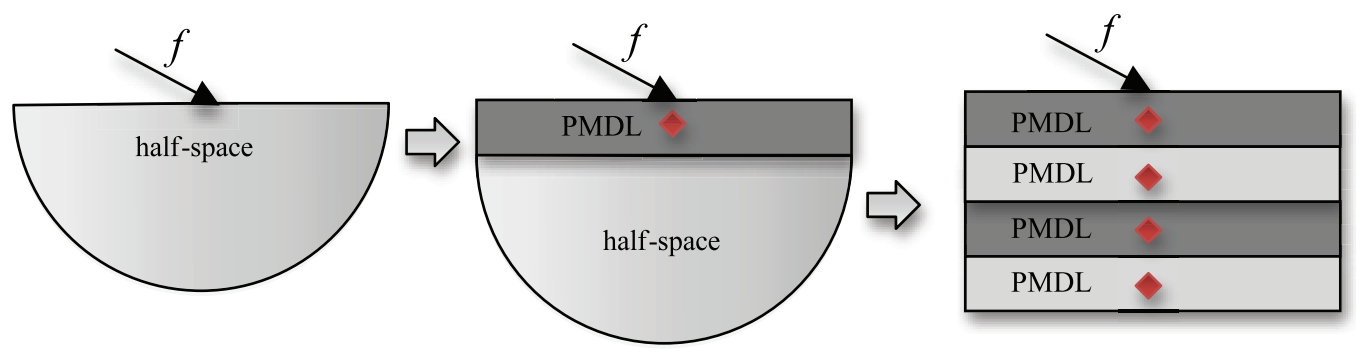

Figure 24. Schematic for PMDL formulation.

The key idea of PMDL method is to discretize the half-space using special linear finite layers to get accurate half-space stiffness. The details are discussed in the following. First, the half-space is replaced with a finite element layer of length $L_{1}$ and another halfspace. The problem set can be defined using following boundary value problem, 


$$
\begin{aligned}
& -\frac{\partial^{2} u}{\partial z^{2}}-k^{2} u=0 \quad \text { for } \mathrm{z} \in\left(z_{0}, \mathrm{z}_{0}+L_{1}\right), \\
& -\frac{\partial u}{\partial z}=f \text { on } \mathrm{z}=z_{0}, \\
& -\frac{\partial u}{\partial z}=\lambda u \quad \text { on } \mathrm{z}=\mathrm{z}_{0}+L_{1} .
\end{aligned}
$$

Multiplying the first equation by virtual wavefield and performing integration by parts, we get:

$$
\int_{z_{0}}^{z_{0}+L_{1}} \frac{\partial \delta u^{*}}{\partial z} \frac{\partial u}{\partial z} d z-\int_{z_{0}}^{z_{0}+L_{1}} \delta u^{*} k^{2} u d z=\left.\delta u^{*} \frac{\partial u}{\partial z}\right|_{z=z_{0}+L_{1}}-\left.\delta u^{*} \frac{\partial u}{\partial z}\right|_{z=z_{0}},
$$

where $\delta u^{*}$ is the virtual wavefield. Using linear shape functions, $N_{1}=x / L_{1}, N_{2}=1-x / L_{1}$ and computing integrals with Gaussian quadrature rule, we get

$$
\left.\left[\begin{array}{ll}
a_{1} & b_{1} \\
b_{1} & a_{1}
\end{array}\right] \begin{array}{l}
u_{0} \\
u_{1}
\end{array}\right\}=\left\{\begin{array}{l}
-\left.\frac{\partial u}{\partial z}\right|_{z=z_{0}} \\
\left.\frac{\partial u}{\partial x}\right|_{z=z_{0}+L_{1}}
\end{array}\right\}
$$

where

$$
a_{1}=\frac{1}{L_{1}}-k^{2} \frac{L_{1}}{4}\left(1+\alpha^{2}\right), b_{1}=-\frac{1}{L_{1}}-k^{2} \frac{L_{1}}{4}\left(1-\alpha^{2}\right),
$$

and $\alpha$ is the coordinate of integration points. Substituting boundary values from problem set (A-5) to Equation (A-7), we get the linear system of equations for the composite half-space

$$
\left[\begin{array}{cc}
a_{1} & b_{1} \\
b_{1} & a_{1}+\lambda
\end{array}\right]\left\{\begin{array}{l}
u_{0} \\
u_{1}
\end{array}\right\}=\left\{\begin{array}{c}
-\frac{\partial u}{\partial z} \\
\left.\right|_{z=z_{0}} \\
0
\end{array}\right\}
$$

Eliminating $u_{1}$, we get,

$$
\left(a_{1}-\frac{b_{1}^{2}}{a_{1}+\lambda}\right) u_{0}=-\left.\frac{\partial u}{\partial z}\right|_{z=z_{0}},
$$

where the exact displacement is recovered if, 


$$
\lambda=a_{1}-\frac{b_{1}^{2}}{a_{1}^{2}+\lambda},
$$

or equivalently

$$
a^{2}-b^{2}=-\lambda^{2}
$$

Examining Equation (A-8) and noting that $\lambda=-i k$, it can be easily shown that the above equality holds only if $\alpha=0$ (i.e. mid-point integration) and length of the finite layer can be an arbitrary number [23]. Since the midpoint integration preserves the half-space stiffness, these finite element layers match in impedance with the exact half-space, even after discretization - thus they are called perfectly matched discrete layers (PMDLs).

Repeating above procedure, we can replace the half-space with infinite number of mid-point integrated layers with arbitrary lengths. However, using infinite number of PMDLs is computationally impossible, and PMDL region is truncated at some point with Dirichlet boundary condition. This truncation causes reflection which results in error in approximation of the half-space stiffness. The accuracy of the approximation can be measured by reflection coefficient given by:

$$
R=\left|\frac{\lambda-\lambda_{\text {approx }}}{\lambda+\lambda_{\text {approx }}}\right|,
$$

and for $n$ midpoint-integrated finite-element layers [16],

$$
R=\prod_{j}^{n}\left(\frac{\left(k-2 i / L_{j}\right.}{k+2 i / L_{j}}\right)^{2},
$$

which indicates that the element lengths must be imaginary or complex for the reflection coefficient to converge to zero. Thus, the half-space can be replaced by finite number of complex-length midpoint-integrated finite elements leading to a good approximation of the stiffness at $z_{0}$ - this is the PMDL approximation.

The approximate half-space stiffness relation can be written by assembling stiffness matrices of PMDL layers as: 


$$
\left\{\begin{array}{c}
-\frac{\partial u_{0}}{\partial z} \\
0 \\
\vdots \\
0
\end{array}\right) \approx\left[\begin{array}{cccc}
a_{1} & b_{1} & & \\
b_{1} & a_{1}+a_{2} & \ddots & \\
& \ddots & \ddots & b_{n-1} \\
& & b_{n-1} & a_{n-1}+a_{n}
\end{array}\right]\left\{\begin{array}{c}
u_{0} \\
u_{a_{1}} \\
\vdots \\
u_{a_{n-1}}
\end{array}\right\},
$$

Where $u_{a_{j}}$ are auxiliary variables. For the discussion in this paper Equation (A-15) is written in a compact form as:

$$
\left\{\begin{array}{c}
\partial u_{0} / \partial z \\
0 \\
0
\end{array}\right\} \approx\left[\begin{array}{lll}
K_{0,0} & K_{0, a_{1}} & \\
K_{a_{1}, 0} & K_{a_{1}, a_{1}} & \mathbf{K}_{a_{1}, a} \\
& \mathbf{K}_{a, a_{1}} & \mathbf{K}_{a, a}
\end{array}\right]\left\{\begin{array}{l}
u_{0} \\
u_{a_{1}} \\
\mathbf{u}_{a}
\end{array}\right\}
$$

where $\mathbf{u}_{a}$ is a vector of auxiliary variables for $j=2,3, \ldots n-1$. Taking inverse Fourier transform of Equation (A-16), we get approximate DtN map for the 2-D Equation (A-1) as:

$$
\left\{\begin{array}{c}
\partial \mathbf{u}_{0} / \partial \mathbf{n} \\
\mathbf{0} \\
\mathbf{0}
\end{array}\right] \approx\left[\begin{array}{lll}
\mathbf{K}_{0,0} & \mathbf{K}_{0, a_{1}} & \\
\mathbf{K}_{a_{1}, 0} & \mathbf{K}_{a_{1}, a_{1}} & \mathbf{K}_{a_{1}, a} \\
& \mathbf{K}_{a, a_{1}} & \mathbf{K}_{a, a}
\end{array}\right]\left[\begin{array}{l}
\mathbf{u}_{0} \\
\mathbf{u}_{a_{1}} \\
\mathbf{u}_{a}
\end{array}\right\} .
$$

We want to emphasize that similar matrix equation can be obtained using other local $\mathrm{ABC}$ methods including PML and rational ABCs which are often implemented using auxiliary variables.

The PMDL parameters $L_{j}$ should be optimized such that the Equation (A-17) becomes an accurate representation of the half-space stiffness relation. For a homogeneous medium, the approximate stiffness of half-space $\boldsymbol{\lambda}_{n}$ (associated with $n$ PMDL layers) is the Schur complement of the matrix in Equation (A-16) with respect to the $K_{0,0}$. Thus,

$$
\lambda_{n}=K_{0,0}-\left[\begin{array}{ll}
K_{0, a_{1}} & \mathbf{0}
\end{array}\right]\left[\begin{array}{ll}
\mathbf{K}_{a_{1}, a_{1}} & \mathbf{K}_{a_{1}, a} \\
\mathbf{K}_{a, a_{1}} & \mathbf{K}_{a, a}
\end{array}\right]^{1}\left[\begin{array}{c}
K_{a_{1}, 0} \\
\mathbf{0}
\end{array}\right],
$$

which is a function of frequency $\omega$ and horizontal wavenumber $k_{x}$. An optimal set of PMDL parameters $L_{j}^{*}$ is the one minimizing the misfit between the approximate stiffness and the 
exact stiffness in Equation (A-4) for a given frequency $\boldsymbol{\omega}$ and a range of horizontal wavenumbers with interval of $\left(k_{x}^{\min }, k_{x}^{\max }\right)$. Specifically:

$$
\min _{L_{j}=L_{j}^{*}}\left|\frac{\lambda_{n}-\lambda}{\lambda}\right| \text { for } \forall k_{x} \in\left(k_{x}^{\min }, k_{x}^{\max }\right)
$$

This optimization process can be carried out using a standard least squares algorithm. Also note that the horizontal wavenumber bounds $k_{x}^{\min }$ and $k_{x}^{\max }$ can be determined based on finite element discretization size and computational domain dimensions (e.g. see [27]).

For a heterogeneous medium, the optimization problem (A-19) must be solved for a range of the velocities, and the optimal set of PMDL parameters minimizes the misfit error over multiple wave velocities. To facilitate the process, we choose a reference wave velocity $c_{0}$ (often the average wave velocity), and we rewrite the PMDL parameters as:

$$
L_{j}=\frac{2 c_{0}}{\omega}\left(\delta_{j}+\gamma_{j} i\right)
$$

where $\left(\boldsymbol{\delta}_{j}, \boldsymbol{\gamma}_{j}\right)$ is the new set of optimization parameters with real values. Figure 25 compares the exact and approximate stiffness of half-space for multiple velocities at frequency of 32 $\mathrm{Hz}$ as a function of horizontal wavenumber. Note that The values are normalized with respect to $\frac{\omega}{c_{0}}$. 


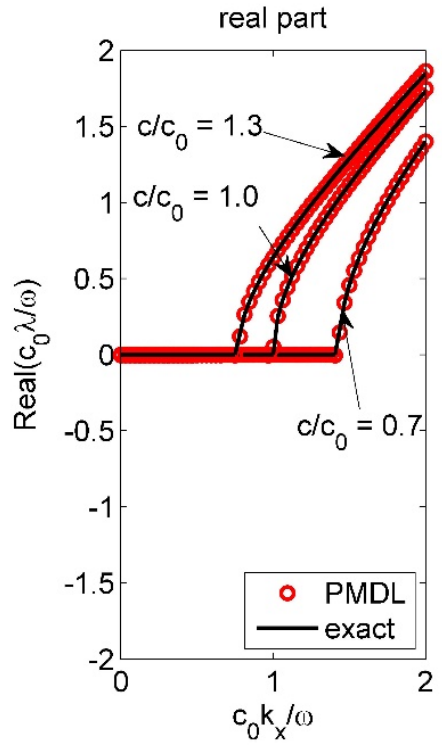

(a)

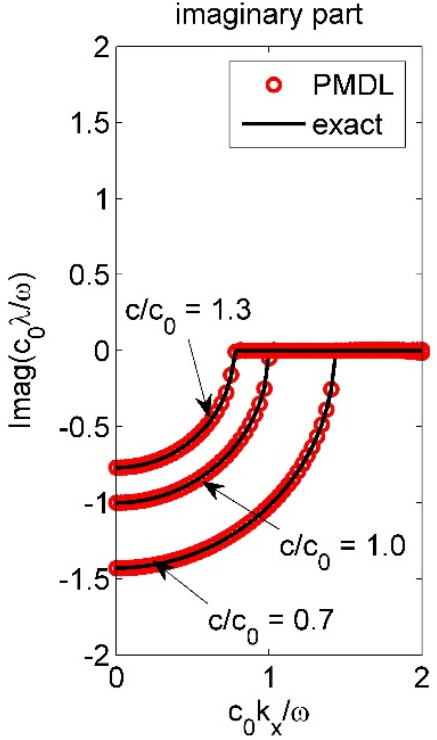

(b)

Figure 25. Comparison of the exact and approximate stiffness of half-space. a) real part, and b) imaginary part. 